\title{
On the Foundations of the Stochastic Immersed Boundary Method
}

\author{
Peter R. Kramer ${ }^{\mathrm{a}, *}$, Charles S. Peskin ${ }^{\mathrm{b}}$, Paul J. Atzberger ${ }^{\mathrm{c}}$ \\ ${ }^{a}$ Department of Mathematical Sciences, Rensselaer Polytechnic Institute, 110 8th \\ St., Troy, NY 12180 \\ ${ }^{\mathrm{b}}$ Courant Institute of Mathematical Sciences, New York University, 251 Mercer \\ St., New York, NY 10012 \\ ${ }^{\mathrm{c}}$ Department of Mathematics, University of California at Santa Barbara, 6712 \\ South Hall, Santa Barbara, CA 93106
}

\begin{abstract}
We explore the theoretical foundations for the inclusion of thermal fluctuations in the immersed boundary method for simulating microscale fluid systems with immersed flexible structures, as in cellular and subcellular biology. We investigate in particular the physical validity of the thermal forcing scheme with respect to the coupling of fluid and immersed structural degrees of freedom and non-equilibrium conditions. We discuss also the shortcomings of a natural alternative scheme in which the thermal fluctuations are applied directly to the structural degrees of freedom through Langevin-type dynamics.
\end{abstract}

Key words: Langevin, thermal fluctuations, fluctuation-dissipation theorem, non-equilibrium

PACS: 47.11.-j, 47.57.-s, 47.61.-k, 47.63.-b, 87.15.Aa, 87.15.Ya, 87.16.Ac

1991 MSC: 60H30, 60H35, 76Z05, 82C31

Stochastic extensions of the Immersed Boundary (IB) Method (Peskin, 2002) to incorporate thermal fluctuations have been developed in Kramer and Peskin (2003); Kramer and Majda (2003/04); Atzberger et al. (2007); Atzberger (2007), with the aim of providing a framework for simulating microscale biological systems such as the exocytosis of vesicles from cells, molecular motors,

* Corresponding author. Tel: +001 (518) 276-6896

Fax: +001 (518) 276-4824

Email address: kramep@rpi.edu (Peter R. Kramer).

URL: www.rpi.edu/ kramep (Peter R. Kramer). 
and physical effects of fluctuations on biomolecules such as DNA. Other simulation approaches, most notably Stokesian/Brownian dynamics (Brady and Bossis, 1988; Sierou and Brady, 2001; Banchio and Brady, 2003; Ermak and McCammon, 1978; Schlick, 2002) and Dissipative Particle Dynamics (Avalos and Mackie, 1997; Español, 1997; Español and Warren, 1995; Hoogerbrugge and Koelman, 1992; Marsh and Yeomans, 1997; Pagonabarraga et al., 1998; Groot and Warren, 1997; Den Otter and Clarke, 2001; Nikunen et al., 2003), have also been designed to simulate microphysical systems. These methods are built upon equations for the particle dynamics, with the effects of the fluid handled in approximate manner such as through the assumption of a quasisteady fluid flow acting on rigid particles in the Stokesian/Brownian dynamics algorithm. On the other hand, the IB Method is a fluid-centered simulation, with the heterogenous system being treated to a first approximation as a single fluid obeying the Navier-Stokes equations, with the force density representing the particle forces and elastic properties of the immersed structures. We shall discuss briefly the nature of the approximations for the fluid-structure coupling in the IB Method in Section 1. Beyond the more accurate accounting of the fluid dynamics, another advantage of a fluid-centered approach is the more natural management of topology conservation of extended objects such as polymers (Atzberger et al., 2007). Other fluid-centered simulation approaches for microphysical systems with thermal fluctuations have been developed for the lattice-Boltzmann method (Ladd, 1994; Ladd and Verberg, 2001), for the lattice gas method (Inoue et al., 2002), and within a finite element framework (Sharma and Patankar, 2004). A hybrid fluid-particle theory with thermal fluctuations which is similar to, but has important distinctions from, the stochastic IB method described here is reviewed in Öttinger and Rabin (1989).

Algorithms for the stochastic IB Method including thermal fluctuations are presented in Kramer and Peskin (2003); Atzberger et al. (2007), and their results compared theoretically and numerically against various basic test problems to demonstrate that fundamental aspects of nonequilibrium statistical mechanics are faithfully represented in the simulation scheme. Some movies and results from the numerical simulation examples can be found at http:// www.math.ucsb.edu/ atzberg/stoch_ib/index.html. Further mathematical analyses of the stochastic IB Method are developed in Kramer and Majda (2003/04); Atzberger (2007) to demonstrate more rigorously that the simulations should exhibit correctly many statistical long-time properties accurately. Particular attention is given in Atzberger et al. (2007) to the design of a scheme which permits long time steps which can even underresolve some or all of the fluid degrees of freedom without sacrificing accuracy in the influence of those fluid modes on the immersed structural dynamics. A quantitative analysis of the accuracy of this long-time stepping scheme is presented in Atzberger and Kramer (2006). In the present work, we will examine several fundamental aspects of the stochastic IB Method which have not been addressed in detail in 
these previous works.

The first issue concerns the choice of the random thermal forces to add to the standard IB equations, reviewed in Section 1, to represent the thermal fluctuations arising from collision with unresolved fluid molecules at finite fixed temperature. A summary of the stochastic IB algorithm, including thermal forces, is presented in Section 2. We remark first that we can retain a continuum-based description of the fluid and the Navier-Stokes equation as a description for the deterministic component of the fluid dynamics, since we are interested in simulating macromolecules and structures considerably larger than the size of a water molecule. The mathematical structure of the forces was motivated in Atzberger et al. (2007) through a statistical mechanical consideration (known as the fluctuation-dissipation theorem (Kubo et al., 1991; Kubo, 1966; Fox and Uhlenbeck, 1970)) of a fluid undergoing thermal fluctuations about a rest state. In Section 3, we extend these considerations, following the method of Fox and Uhlenbeck (1970), of near-equilibrium thermal fluctuations of the coupled system of the fluid and immersed structures, and show that the thermal forcing structure deduced in Atzberger et al. (2007) carries over unchanged when the immersed structural dynamics are coupled to the fluid. This statement is nontrivial and relies on some special properties for how the fluid and immersed structures are coupled in the IB Method. The modifications to the approach to take into account the spatial discretization of the numerical scheme are presented in Section 4. We briefly argue in Section 5 that the thermal forcing scheme deduced from a near-equilibrium theory ought also be applicable, without change, to a rather broad range of far-from-equilibrium conditions. We proceed next in Section 6 to a brief consideration of some technical considerations regarding restrictions on the time step for numerical accuracy.

Through the procedure outline above, we will find that the principles of statistical mechanics provide a rather specific statistical structure for the thermal forces, so that no further need for modeling approximations is necessary. This derivation, though, is premised on the assertion that the deterministic structure of the IB equations should be preserved on the microscale. An approach motivated by stochastic models for Brownian motion (Pathria, 1972; Nelson, 1967; Kramer, 2005; Reichl, 1998) might, by contrast, begin by modifying the equations for the dynamics of the immersed structures, which are conservative in the standard IB framework, through the incorporation of a friction term which allows the immersed particles to slip relative to the local fluid velocity (Oster and Peskin, 1992). Applying the statistical mechanical procedure to such a system would give rise to direct thermal forcing of the structural degrees of freedom (with close analogies to Langevin particle dynamics (Pathria, 1972; Nelson, 1967; Reichl, 1998)), in contrast to the thermal forcing structure of the stochastic IB method we are advancing, in which the fluctuation-dissipation theorem of statistical mechanics indicates that thermal forcing should be ap- 
plied only to the fluid degrees of freedom. While the inclusion of thermal forces directly on the particles may appear more intuitive and more parallel to other developments (such as Stokesian/Brownian dynamics (Brady, 1993; Brady and Bossis, 1988; Ermak and McCammon, 1978)), we will explain in Section 7 how this approach is physically problematic in the context of the IB Method.

\section{Deterministic Immersed Boundary Method Equations}

In the standard implementations of the IB method, the entire fluid-particle system is treated as a constant density fluid. We moreover assume the fluid volume $\Omega$ is a cube of side length $L$ with periodic boundary conditions, as is typical for applications of the IB method, because it permits the use of a Fast Fourier Transform Peskin and McQueen (1995); Peskin (2002).

The evolution of this fluid is usually given by the incompressible Navier-Stokes equations, but we will neglect the nonlinear advection term in the present work since thermal fluctuations are usually relevant only for microscale systems at low Reynolds number:

$$
\begin{aligned}
\rho \frac{\partial \vec{u}(\vec{x}, t)}{\partial t} & =\mu \nabla^{2} \vec{u}(\vec{x}, t)-\nabla p(\vec{x}, t)+\vec{f}(\vec{x}, t) \\
\nabla \cdot \vec{u}(\vec{x}, t) & =0
\end{aligned}
$$

where $\vec{u}(\vec{x}, t)$ is the fluid velocity, $\rho$ is the density, $\mu$ is the dynamic viscosity, $p$ is the pressure, and $\vec{f}(\vec{x}, t)$ is a force density. We suppose that the only force acting on the fluid are those associated with forces acting on the immersed structures, represented as a collection of discrete elementary particles with certain force laws between them. We remark that even extended structures such as polymers and membranes can be represented readily within this framework through an appropriate discretization into elementary particles with appropriate force laws connecting them (Atzberger et al., 2007). Each of these forces acting on the structures will be spread near the particle locations $\left\{\vec{X}^{(j)}(t)\right\}_{j=1}^{N}$ via a delta function $\delta_{a}(\vec{x})$ smoothed over a length scale $a$ and possessing good numerical properties Peskin and McQueen (1995). We therefore write for the force density induced by the immersed structures:

$$
\vec{f}(\vec{x}, t)=-\sum_{j=1}^{N} \nabla_{j} \Phi\left(\left\{\vec{X}^{(j)}(t)\right\}_{j=1}^{N}\right) \delta_{a}\left(\vec{x}-\vec{X}^{(j)}(t)\right)
$$

where $\Phi\left(\left\{\vec{x}^{(j)}\right\}_{j=1}^{N}\right)$ is the total potential describing the forces on all the particles due to interaction and/or external influences. The symbol $\boldsymbol{\nabla}_{j}$ denotes a 
gradient with respect to the coordinate $\vec{x}^{(j)}$. Note that $a$ acts as an effective particle size.

Even though the Reynolds number is assumed small, we do not discard the time derivative in (1a) and thereby use creeping flow equations because that would require a further assumption that the fluid density is small compared to the density of the immersed structures (Deutch and Oppenheim, 1987), which is not generally a good approximation in physiological systems for which the IB method is designed. The mathematical origin of this further restriction lies in the dependence of the force density on the position of the immersed structures. When the density of the immersed structures is not much larger than those of the fluid, then their inertia is comparable to or smaller than that of the fluid motion, so the quasi-steady flow assumptions become less secure.

The elementary particle positions are updated by simple advection by the fluid at a locally interpolated fluid velocity:

$$
\frac{\mathrm{d} \vec{X}^{(j)}(t)}{\mathrm{d} t}=\vec{u}_{a}\left(\vec{X}^{(j)}(t), t\right) .
$$

The same smoothed delta function that was used to spread force is used to interpolate velocity:

$$
\vec{u}_{a}(\vec{x}, t)=\int_{\Omega} \vec{u}\left(\vec{x}^{\prime}, t\right) \delta_{a}\left(\vec{x}-\vec{x}^{\prime}\right) \mathrm{d} \vec{x}^{\prime}
$$

We will take this system of equations as the basis onto which we wish to add thermal fluctuations in a physically meaningful way. In fact, as we shall see, the fluctuation dissipation theorem from statistical mechanics prescribes how most of the degrees of freedom of the system should be thermally forced, given the structure of the deterministic terms. We will be able to calculate these thermal forcing terms in an exact fashion, but we pause to mention that the deterministic system (1) on which they are built is not physically exact because of two simplifications of fluid-particle coupling in the IB Method:

(1) The elementary particle motion is treated by advection at a local fluid velocity, rather than by dynamical acceleration taking into account the stress exerted by the fluid on the particle interface.

(2) The influence of the elementary particles on the fluid is represented through force-spreading rather than through a detailed condition, such as no-slip, on a physical particle boundary.

The reason for these simplifications is, of course, to allow the method to handle larger systems over longer time scales than expensive physically precise simulation methods (Glowinski et al., 2001; Patankar et al., 2000; Karrila et al., 
1989; Tran-Cong and Phan-Thien, 1989) can. These assumptions have been demonstrated to work well on the macroscale (Peskin, 2002). Other methods, such as Stokesian dynamics (Brady and Bossis, 1988; Sierou and Brady, 2001; Banchio and Brady, 2003; Ermak and McCammon, 1978; Schlick, 2002) and dissipative particle dynamics (Avalos and Mackie, 1997; Español, 1997; Español and Warren, 1995; Hoogerbrugge and Koelman, 1992; Marsh and Yeomans, 1997; Pagonabarraga et al., 1998; Groot and Warren, 1997; Den Otter and Clarke, 2001; Nikunen et al., 2003) may handle these aspects of the fluidparticle coupling more realistically but make other physical approximations, such as creeping flow, which may not be fully appropriate for physiological systems with comparable density in the fluid and immersed structures.

We note the approximations in the standard IB method because they raise the question of whether an "exact" thermal forcing scheme for a physical approximation to reality will give statistical properties which are approximations to real statistical properties of thermally fluctuating fluid-structure systems. The answer is not a priori clear, but separate analysis and numerical simulations (Kramer and Majda, 2003/04; Atzberger et al., 2007; Atzberger, 2006) give evidence for a substantially affirmative answer.

\section{Summary of Stochastic Immersed Boundary Method}

We will use the standard spatial setup (Peskin and McQueen, 1995) of the IB equations (1), in which the fluid domain $\Omega$ is taken to be a periodic cube with length $L$ in each coordinate direction. The velocity field $\vec{u}$ and the pressure $p$ are discretized on a periodic grid $(\Delta x) \mathbb{Z}_{K}^{3}$, where

$$
\mathbb{Z}_{K}^{3} \equiv[1,2, \ldots, K]^{3},
$$

so that $K$ grid points with grid spacing $\Delta x=L / K$ span the period in each coordinate direction. The positions of the elementary particles $\left\{\vec{X}^{(j)}\right\}_{j=1}^{N}$, however, can take values anywhere within the continuous fluid domain $\Omega$. Time is discretized into intervals of uniform size $\Delta t$, and the discretized variables will be represented with a superscript denoting the time step.

In Atzberger et al. (2007), we develop the following thermal forcing scheme for the IB method, where the time step $\Delta t$ need not necessarily resolve the 
dynamics of the fluid variables:

$$
\begin{aligned}
\vec{u}^{n+1} & =\mathrm{P}\left[\mathrm{e}^{\mathcal{M} \Delta t} \vec{u}^{n}+\rho^{-1} \int_{0}^{\Delta t} \mathrm{e}^{\mathcal{M}(\Delta t-t)} \vec{f}^{n}+\vec{\Xi}_{T}^{n}\right], \\
\vec{f}^{n}(\vec{x}) & =-\sum_{j=1}^{N} \nabla_{j} \Phi\left(\left\{\vec{X}_{j}^{n}\right\}\right) \delta_{a}\left(\vec{x}-\vec{X}_{j}^{n}\right), \\
\overline{\vec{u}}^{n} & =\frac{1}{\Delta t} \int_{0}^{\Delta t}\left[\mathrm{e}^{\mathcal{M} t} \vec{u}^{n}+\rho^{-1} \mathrm{P} \int_{0}^{t} \mathrm{e}^{\mathcal{M}\left(t-t^{\prime}\right)} \vec{f}^{n} \mathrm{~d} t^{\prime}\right] \mathrm{d} t \\
& \quad+\mathrm{P} \vec{G}^{n} \\
\vec{X}_{j}^{n+1} & =\vec{X}_{j}^{n}+\sum_{\vec{x} \in(\Delta x) \mathbb{Z}_{K}^{3}} \overline{\vec{u}}^{n}(\vec{x}) \delta_{a}\left(\vec{x}-\vec{X}_{j}^{n}\right)(\Delta x)^{3} \Delta t .
\end{aligned}
$$

We have presented the scheme here concisely in terms of operators: $\mathcal{M}=\nu L_{\Delta x}^{0}$ with a standard center difference approximation for the Laplacian is the viscous dissipation operator (expressed in terms of the kinematic viscosity $\nu=\mu / \rho)$, and $\mathbf{P}$ is the operator projecting onto the space of functions satisfying incompressibility with respect to the discrete central-difference divergence operator: $\vec{D}_{\Delta x}^{0} \cdot \vec{u}=0$. Part of the procedure for allowing a time step which underresolves the fluid is the calculation of the fluid velocity averaged over a time step, $\overline{\vec{u}}^{n}$. Its thermal driving $\vec{G}^{n}$ is naturally correlated with the thermal driving $\vec{\Xi}_{T}^{n}$ which updates the fluid velocity from the beginning to the end of a time step.

For the usual periodic cubic domain, the above operators and functions are most conveniently implemented with respect to a Fourier series expansion for the fluid velocity

$$
\vec{u}^{n}(\vec{x})=\sum_{\vec{k} \in \mathbb{Z}_{K}^{3}} \hat{\vec{u}}_{\vec{k}}^{n} \mathrm{e}^{2 \pi \mathrm{i} \vec{k} \cdot \vec{x}}
$$

and affiliated physical fields with similar notation. The operators $\mathcal{M}$ and $\mathrm{P}$ are block diagonal with respect to these Fourier expansions:

$$
\hat{\mathcal{M}}_{\vec{k}, \vec{k}^{\prime}}=-\alpha_{\vec{k}} \delta_{\vec{k}, \vec{k}^{\prime}}, \quad \hat{\mathrm{P}}_{\vec{k}, \vec{k}^{\prime}}=\left(\mathrm{I}-\frac{\hat{\vec{g}}_{\vec{k}} \otimes \hat{\vec{g}}_{\vec{k}}}{\left|\hat{\vec{g}}_{\vec{k}}\right|^{2}}\right) \delta_{\vec{k}, \vec{k}^{\prime}}
$$

where

$$
\begin{aligned}
\alpha_{\vec{k}} & =\frac{2 \mu}{\rho(\Delta x)^{2}} \sum_{j=1}^{3}\left(1-\cos \left(2 \pi k_{j} / K\right)\right), \\
\hat{\vec{g}}_{\vec{k}, j} & =\frac{1}{\Delta x} \sin \left(2 \pi k_{j} / K\right),
\end{aligned}
$$

I is the identity matrix, and $\delta_{\vec{k}, \vec{k}^{\prime}}$ is the Kronecker delta function (which equals one when $\vec{k}=\vec{k}^{\prime}$ and is zero otherwise). The Fourier coefficients of the thermal 
driving are computed as follows:

$$
\vec{\Xi}_{T, \vec{k}}^{n}=\sigma_{\vec{k}} \tilde{\vec{Z}}_{\vec{k}}^{n}, \quad \quad \vec{G}_{\vec{k}}^{n}=c_{1, \vec{k}} \vec{\Xi}_{T, \vec{k}}^{n}+c_{2, \vec{k}} \stackrel{\vec{Y}}{\vec{k}}^{n}
$$

where $\left\{\tilde{\vec{Z}}_{\vec{k}}^{n}, \tilde{\vec{Y}}_{\vec{k}}^{n}\right\}_{\vec{k} \in \mathbb{Z}_{K}^{3}, n=0,1,2, \ldots}$ are collections of complex vector-valued Gaussian random variables, all real and imaginary components of which have mean zero with variance 1 and are mutually independent from all other components with the exception of a complex conjugacy constraint: $\tilde{\vec{Z}}_{-\vec{k}}^{n}=\overline{\tilde{\vec{Z}}_{\vec{k}}^{n}}$ and $\tilde{\overrightarrow{\vec{Y}}}_{-\vec{k}}^{n}=\overline{\tilde{\vec{Y}}_{\vec{k}}^{n}}$ with $-\vec{k}$ to be interpreted as reflection across the origin in the periodic lattice $\mathbb{Z}_{K}^{3}$. The thermal noise amplitudes are given by the formulas:

$$
\begin{aligned}
\sigma_{\vec{k}}^{2} & =\frac{A_{\vec{k}} k_{B} T}{2 \rho L^{3}}\left(1-\mathrm{e}^{-2 \alpha_{\vec{k}} \Delta t}\right) \\
c_{1, \vec{k}} & =\frac{1}{\alpha_{\vec{k}} \Delta t} \tanh \left(\frac{\alpha_{\vec{k}} \Delta t}{2}\right), \\
c_{2, \vec{k}} & =\sqrt{\left[\frac{A_{\vec{k}} k_{B} T}{\rho L^{3} \alpha_{\vec{k}}^{2}(\Delta t)^{2}}\right]\left[\alpha_{\vec{k}} \Delta t-2 \tanh \left(\frac{\alpha_{\vec{k}} \Delta t}{2}\right)\right] .}
\end{aligned}
$$

where

$$
\begin{aligned}
A_{\vec{k}} & = \begin{cases}2, & \vec{k} \in \mathcal{K}, \\
1, & \vec{k} \notin \mathcal{K},\end{cases} \\
\mathcal{K} & =\left\{\vec{k}: k_{j}=0 \text { or } k_{j}=N / 2, j=1,2,3\right\} .
\end{aligned}
$$

A detailed algorithmic description for this simulation scheme is presented in Atzberger et al. (2007).

\section{Derivation of Thermal Driving for Immersed Boundary Equa- tions in Continuum Limit}

We now proceed to show how the fluctuation-dissipation theorem (in the form developed in Fox and Uhlenbeck (1970)) provides an essentially complete and explicit specification for the appropriate thermal fluctuations for the coupled equations for the fluid and immersed structures in the IB Method. The general ingredients are the product of the temperature and Boltzmann's constant $k_{B} T$, the energy as a functional of the physical variables, and the deterministic structure of the equations of motion.

The application of the fluctuation-dissipation theorem, in the form (Fox and Uhlenbeck, 1970) we will use in the present work, amounts precisely to the following: 
(1) Assume the thermal forces are delta-correlated in time, modeling rapid molecular-scale fluctuations.

(2) Linearize the equations of motion about equilibrium, and expand the energy functional to quadratic order about equilibrium.

(3) Compute the correlation structure between the forces acting on the various variables which guarantees that when the system achieves a statistically stationary state (thermal equilibrium), then the fluctuations of the system variables obey the Gibbs-Boltzmann distribution.

As we shall discuss at a little more length in Section 5, the thermal fluctuation structure prescribed by the fluctuation-dissipation theorem also applies in nonequilibrium situations so long as the fluctuations do not produce significant spatial variations in the temperature and density of the fluid Español (1998). For physiological conditions, it can be checked that temperature and density fluctuations are appreciable only on the nanometer scales, which we would never attempt to resolve with the IB method.

To maintain simplicity with the formal calculations, we begin with an application of the fluctuation-dissipation theorem to derive the appropriate thermal driving structure for the continuum equations of the IB method, summarized in Section 1, on a domain with period $L$ in each direction. We take the standard equations as providing the deterministic structure, and add random driving terms to each equation to account for the effects of thermal fluctuations:

$$
\begin{aligned}
& \frac{\mathrm{d} \vec{X}^{(j)}(t)}{\mathrm{d} t}=\vec{u}_{a}\left(\vec{X}^{(j)}(t), t\right)+\vec{V}_{r}^{(j)}(t), \\
& \rho \frac{\partial \vec{u}(\vec{x}, t)}{\partial t}=\mu \nabla^{2} \vec{u}(\vec{x}, t)-\nabla p(\vec{x}, t)+\vec{f}_{r}(\vec{x}, t) \\
& -\sum_{j=1}^{N} \boldsymbol{\nabla}_{j} \Phi\left(\left\{\vec{X}^{(j)}(t)\right\}\right) \delta_{a}\left(\vec{x}-\vec{X}^{(j)}(t)\right), \\
& \boldsymbol{\nabla} \cdot \vec{u}(\vec{x}, t)=0 .
\end{aligned}
$$

Note that the first equation is not a dynamical equation; the random driving $\vec{V}_{r}^{(j)}(t)$ amounts to the introduction of random velocities not random forces. One might object that such random driving is non-physical; note however that such random driving terms appear routinely in turbulent advection models to represent the effects of molecular diffusion (Majda and Kramer, 1999) and does appear in the Coupled Langevin Equations approach (Öttinger and Rabin, 1989), which has some similarities to the stochastic IB method but differs in some fundamental aspects with regard to the coupling of the fluid and immersed structures. In any case, we will find that the fluctuation-dissipation theorem insists that $\vec{V}_{r}^{(j)}(t) \equiv 0$ for this system.

We now proceed to consider first small fluctuations about equilibrium, and 
assume the random drivings are delta-correlated in time. Then we will use the fluctuation-dissipation theorem Fox and Uhlenbeck (1970) to show that the correct form for the random driving is:

$$
\begin{aligned}
\vec{V}_{r}^{(j)}(t) & \equiv 0, \\
\vec{f}_{r}(\vec{x}, t) & =\sum_{\vec{k} \in \mathbb{Z}^{3}} \vec{f}_{r, \vec{k}}(t) \mathrm{e}^{2 \pi \mathrm{i} \vec{k} \cdot \vec{x} / L}, \\
\vec{f}_{r, \vec{k}}(t) & =2 \pi k \sqrt{\frac{\mu k_{B} T}{L^{5}}} \tilde{\vec{Z}}_{\vec{k}}(t),
\end{aligned}
$$

where $\left\{\tilde{\vec{Z}}_{\vec{k}}(t)\right\}_{\vec{k} \in \mathbb{Z}^{3}}$ are standard complex white noise processes, the real and imaginary components of which are each mutually independent standard real white noise processes, except for a complex conjugacy constraint:

$$
\tilde{\vec{Z}}_{-\vec{k}}(t)=\overline{\tilde{\vec{Z}}_{\vec{k}}(t)}
$$

A standard real vector-valued white noise $\vec{Z}(t)$ is taken here to indicate a mean zero, Gaussian random process with second order correlation function:

$$
\left\langle\vec{Z}(t) \otimes \vec{Z}\left(t^{\prime}\right)\right\rangle=\mid \delta\left(t-t^{\prime}\right),
$$

where $\mathrm{I}$ is the identity matrix. Note that the $\vec{k}=\overrightarrow{0}$ mode must have zero imaginary component due to the complex conjugacy constraint.

We show now how the thermal forces are derived by insisting that they fluctuate on a microscopic time-scale and are consistent with the Gibbs-Boltzmann distribution for small fluctuations about equilibrium. (These properties together are equivalent to the application of the fluctuation-dissipation theorem.) Since microscopic time scales are taken to zero in a continuum hydrodynamic limit, the thermal forces on the equations should be delta-correlated in time. Parameterizing the thermal forcing of the velocity field by its Fourier coefficients (6c), we therefore write

$$
\begin{aligned}
\left\langle\vec{f}_{r, \vec{k}}(t) \otimes \overrightarrow{\left.f_{r, \vec{k}^{\prime}}\left(t^{\prime}\right)\right\rangle}\right. & =\mathrm{F}_{\vec{k} \vec{k}^{\prime}}^{(f f)} \delta\left(t-t^{\prime}\right), \\
\left\langle\vec{f}_{r, \vec{k}}(t) \otimes \vec{V}_{r}^{(j)}\left(t^{\prime}\right)\right\rangle & =\mathrm{F}_{\vec{k} j}^{(f p)} \delta\left(t-t^{\prime}\right), \\
\left\langle\vec{V}_{r}^{(j)}(t) \otimes \vec{V}_{r}^{\left(j^{\prime}\right)}\left(t^{\prime}\right)\right\rangle & =\mathrm{F}_{j j^{\prime}}^{(p p)} \delta\left(t-t^{\prime}\right),
\end{aligned}
$$

where the matrices $\mathrm{F}^{(f f)}, \mathrm{F}^{(f p)}$, and $\mathrm{F}^{(p p)}$ are to be determined by application of our third criterion that small thermal fluctuations about equilibrium should obey the Gibbs-Boltzmann distribution. We define here the matrix $\mathrm{F}^{(f f)}$ to be a matrix of block $3 \times 3$ matrices $\mathrm{F}_{\vec{k} \vec{k}^{\prime}}^{(f f)}$, with these blocks indexed by the discrete but infinite set of wavenumbers $\left\{\vec{k}, \vec{k}^{\prime} \in \mathbb{Z}^{3}\right\} . \mathrm{F}^{(f f)}$ is then really an infinite-dimensional matrix, but we work formally with it just as with finitedimensional matrices. We will revisit the calculation we are now performing 
for the discretized IB equations in Section 4 where the analogous matrices are finite. The present calculation should just be viewed as a formal indication of what to expect in the continuous limit, though we acknowledge that a rigorous justification would require further scrutiny of the contributions from wavenumbers of large magnitude. Similar remarks apply to the matrices $\mathbf{F}^{(f p)}$ and $\mathrm{F}^{(p p)}$.

We now proceed to determine the instantaneous correlations between the system variables (fluid and particle degrees of freedom) in thermal equilibrium induced by the dynamics linearized about mechanical equilibrium and driven by some as-yet-unspecified thermal terms with correlation structure (8). We will thereby obtain equations for the correlation matrices of the system variables in terms of the correlation matrices of the driving terms. The Gibbs-Boltzmann distribution of statistical mechanics prescribes what the correlation matrices of the system variables should be in terms of the temperature, and in this way, we obtain equations for the correlation matrices $\mathrm{F}^{(f f)}, \mathrm{F}^{(f p)}$, and $\mathrm{F}^{(p p)}$, the solution of which will be shown to be Eq. (6).

We stress the distinction between the notions of mechanical and thermal equilibrium which will be both used throughout this section. Mechanical equilibrium of course refers to system configurations which minimize (or more generally extremize) the energy (Hamiltonian) function of the system. Thermal equilibrium corresponds to a statistically stationary state in which the system variables are fluctuating in time, but their statistics have achieved steady-state values (the computation of which is the domain of equilibrium statistical mechanics). Also, our use of the phrase "correlation" will correspond to that used in stochastic process and random field literature (Yaglom, 1987) rather than in probability theory, where correlations are distinguished from covariances through normalization factors.

To illustrate the main ideas we first consider the case of a unique equilibrium for the immersed structures and then generalize the calculation for the case in which a unique equilibrium does not exist due to symmetries in the en-

ergy functional. We will use the notational convention that $\vec{X}=\left\{\vec{X}^{(j)}\right\}_{j=1}^{N}$ represents a grand vector of all elementary particle positions

\subsection{Case of Unique Equilibrium}

We begin by supposing that the potential energy $\Phi(\vec{X})$ has a unique nondegenerate minimum at $\vec{X}=\vec{X}_{0}$, with nondegeneracy meaning that its Hessian is positive definite at this minimum. Then the fluid-particle system has a unique 
equilibrium

$$
\vec{u}(\vec{x}, t)=0, \quad \vec{X}^{(j)}=\vec{X}_{0}^{(j)} \text { for } j=1, \ldots, N
$$

3.1.0.1 Linearized Dynamics We examine weak thermal fluctuations about equilbrium by linearizing Eq. (6) about equilibrium

$$
\begin{aligned}
& \frac{\mathrm{d} \vec{X}^{(j)}(t)}{\mathrm{d} t}=\vec{u}_{a}\left(\vec{X}_{0}^{(j)}, t\right)+\vec{V}_{r}^{(j)}(t), \\
& \rho \frac{\partial \vec{u}(\vec{x}, t)}{\partial t}=\mu \nabla^{2} \vec{u}(\vec{x}, t)-\nabla p(\vec{x}, t)+\vec{f}_{r}(\vec{x}, t) \\
& -\sum_{j=1}^{N} \sum_{j^{\prime}=1}^{N} \mathrm{H}_{j j^{\prime}}^{(0)} \cdot\left(\vec{X}^{\left(j^{\prime}\right)}(t)-\vec{X}_{0}^{\left(j^{\prime}\right)}\right) \delta_{a}\left(\vec{x}-\vec{X}_{0}^{(j)}\right) \\
& \nabla \cdot \vec{u}(\vec{x}, t)=0,
\end{aligned}
$$

where

$$
\mathrm{H}_{j, j^{\prime}}^{(0)}=\left.\nabla_{j} \nabla_{j^{\prime}} \Phi\left(\left\{\vec{x}^{(j)}\right\}_{j=1}^{N}\right)\right|_{\left\{\vec{x}^{(j)}=\vec{x}_{0}^{(j)}\right\}_{j=1}^{N}} .
$$

Taking now a Fourier transform of these linearized equations, we obtain

$$
\begin{gathered}
\frac{\mathrm{d} \vec{X}^{(j)}(t)}{\mathrm{d} t}=\sum_{\vec{k} \in \mathbb{Z}^{3}} \hat{\vec{u}}_{a, \vec{k}}(t) \mathrm{e}^{2 \pi \mathrm{i} \vec{k} \cdot \vec{X}_{0}^{(j)} / L}+\vec{V}_{r}^{(j)}(t), \\
\frac{\partial \hat{\vec{u}}_{\vec{k}}(t)}{\partial t}=-4 \pi^{2} \nu|\vec{k}|^{2} L^{-2} \mathrm{P}_{\vec{k}} \hat{\overrightarrow{\vec{u}}}_{\vec{k}}(t) \\
-\rho^{-1} \mathrm{P}_{\vec{k}} \sum_{j=1}^{N} \sum_{j^{\prime}=1}^{N} \mathrm{H}_{j j^{\prime}}^{(0)} \cdot\left(\vec{X}^{\left(j^{\prime}\right)}(t)-\vec{X}_{0}^{\left(j^{\prime}\right)}\right) \\
\quad \times \hat{\delta}_{a, \vec{k}} \mathrm{e}^{-2 \pi \mathrm{i} \vec{k} \cdot \vec{X}_{0}^{(j)} / L} \\
+\rho^{-1} \mathrm{P}_{\vec{k}} \vec{f}_{r, \vec{k}}(t) .
\end{gathered}
$$

Some new notation has been introduced here:

- the Fourier coefficients of the interpolated velocity field are obtained from Eq. (1d) and the convolution theorem for Fourier series:

$$
\hat{\vec{u}}_{a, \vec{k}}(t)=L^{3} \hat{\vec{u}}_{\vec{k}}(t) \hat{\delta}_{a, \vec{k}}
$$

where $\hat{\delta}_{a, \vec{k}}$ is to be understood as the Fourier coefficient of a periodized extension of the delta function $\delta_{a}$, 
- the tensor which enforces incompressibility by projecting Fourier vector coefficients transversely to the wavevector :

$$
\mathrm{P}_{\vec{k}} \equiv \mathrm{I}-\frac{\vec{k} \otimes \vec{k}}{|\vec{k}|^{2}}
$$

where $\mathrm{I}$ is the identity matrix.

In proceeding, we will find it convenient to define a single high-dimensional "system vector" which comprises the deviation of the fluid and particle variables from their equilibrium values:

$$
\vec{a}(t)=\left[\begin{array}{c}
\left\{\hat{\vec{u}}_{\vec{k}}(t)\right\}_{\vec{k} \in \mathbb{Z}^{3}} \\
\left\{\vec{X}^{(j)}(t)-\vec{X}_{0}^{(j)}\right\}_{j=1}^{N}
\end{array}\right] .
$$

We note that the fluid mode variables must satisfy the conjugacy relations:

$$
\hat{\vec{u}}_{\vec{k}}=\overline{\hat{\vec{u}}_{-\vec{k}}} \text {. }
$$

Then we can write Eq. (10c) as an abstract linear evolution equation:

$$
\frac{\mathrm{d} \vec{a}(t)}{\mathrm{d} t}=\mathrm{D} \vec{a}(t)+\vec{g}(t)
$$

where the terms on the right hand side are defined as follows:

- The constant matrix D can be expressed in block form

$$
\mathrm{D}=\left[\begin{array}{cc}
\mathrm{D}^{(f f)} & \mathrm{D}^{(f p)} \\
\mathrm{D}^{(p f)} & 0
\end{array}\right]
$$

with the blocks defined:

$$
\begin{aligned}
\mathrm{D}_{\vec{k}, \vec{k}^{\prime}}^{(f f)} & =-4 \pi^{2} \nu|\vec{k}|^{2} L^{-2} \mathrm{P}_{\vec{k}} \delta_{\vec{k}, \vec{k}^{\prime}} \\
\mathrm{D}_{\vec{k}, j^{\prime}}^{(f p)} & =-\rho^{-1} \mathrm{P}_{\vec{k}} \sum_{j=1}^{N} \mathrm{H}_{j j^{\prime}}^{(0)} \hat{\delta}_{a, \vec{k}} \mathrm{e}^{-2 \pi \mathrm{i} \vec{k} \cdot \vec{X}_{0}^{(j)} / L}, \\
\mathrm{D}_{j, \vec{k}^{\prime}}^{(p f)} & =L^{3} \hat{\delta}_{a, \vec{k}^{\prime}} \mathrm{e}^{2 \pi \mathrm{i} \vec{k}^{\prime} \cdot \vec{X}_{0}^{(j)} / L} .
\end{aligned}
$$

- The thermal driving system vector is defined in a similar manner to the system vector $\vec{a}(t)$ in Eq. (11):

$$
\vec{g}(t)=\left[\begin{array}{c}
\rho^{-1}\left\{\mathrm{P}_{\vec{k}} \vec{f}_{r, \vec{k}}(t)\right\}_{\vec{k} \in \mathbb{Z}^{3}} \\
\left\{\vec{V}_{r}^{(j)}(t)\right\}_{j=1}^{N}
\end{array}\right],
$$


with correlation function

$$
\begin{aligned}
\left\langle\vec{g}(t) \otimes \overline{\left.\vec{g}\left(t^{\prime}\right)\right\rangle}\right. & =\mathrm{G} \delta\left(t-t^{\prime}\right), \\
\mathrm{G} & =\left[\begin{array}{cc}
\rho^{-2} \mathrm{PF}^{(f f)} \mathrm{P}^{\dagger} & \rho^{-1} \mathrm{PF}^{(f p)} \\
\rho^{-1} \mathrm{~F}^{(p f)} \mathrm{P}^{\dagger} & \mathrm{F}^{(p p)}
\end{array}\right],
\end{aligned}
$$

where

$$
(\mathrm{P})_{\vec{k}, \vec{k}^{\prime}}=\mathrm{P}_{\vec{k}} \delta_{\vec{k}, \vec{k}^{\prime}}
$$

The blocks in $\mathrm{G}$ are the correlation structures of thermal driving on the fluid and particle variables and are defined through Eq. (8).

3.1.0.2 System Variable Correlations in Thermal Equilibrium Our objective is to derive the form of $\mathrm{G}$ which will induce the correct statistics for the system variables $\vec{a}(t)$ in thermal equilibrium. We proceed now to describe the mathematical expression for the thermal equilibrium statistics of $\vec{a}(t)$. The energy of the IB system is

$$
E(\vec{u}, \vec{X})=\frac{1}{2} \rho \int_{\Omega}|\vec{u}|^{2}+\Phi(\vec{X})=\frac{1}{2} M \sum_{\vec{k} \in \mathbb{Z}^{3}}\left|\hat{\vec{u}}_{\vec{k}}\right|^{2}+\Phi(\vec{X})
$$

where $M=\rho L^{3}$ is the total mass of the fluid in the periodic domain. For small fluctuations about equilibrium we take a quadratic expansion of the energy about its minimum:

$$
\begin{aligned}
E(\vec{u}, \vec{X}) & \approx E_{\text {app }}\left(\left\{\hat{\vec{u}}_{\vec{k}}\right\}, \vec{X}\right) \\
& =\frac{1}{2} M \sum_{\vec{k} \in \mathbb{Z}^{3}}\left|\hat{\vec{u}}_{\vec{k}}\right|^{2} \\
& +\frac{1}{2} \sum_{j=1}^{N} \sum_{j^{\prime}=1}^{N}\left(\vec{X}^{(j)}-\vec{X}_{0}^{(j)}\right) \cdot \mathrm{H}_{j, j^{\prime}}^{(0)} \cdot\left(\vec{X}^{\left(j^{\prime}\right)}-\vec{X}_{0}^{\left(j^{\prime}\right)}\right) \\
& \equiv \frac{1}{2} \vec{a} \cdot \mathrm{E} \cdot \overrightarrow{\vec{a}}
\end{aligned}
$$

where

$$
\mathrm{E}=\left[\begin{array}{cc}
M \mathrm{I} & 0 \\
0 & \mathrm{H}^{(0)}
\end{array}\right]
$$

The Gibbs-Boltzmann distribution describes the fluctuations of the system 
variables in thermal equilibrium:

$$
\begin{aligned}
& \operatorname{Prob}(\{\vec{u}, \vec{X}\} \in A)= \\
& Z^{-1} \int_{A} \exp \left[-E(\{\vec{u}, \vec{X}\}) /\left(k_{B} T\right)\right] \\
& \prod_{\vec{k}^{\prime} \in \mathbb{Z}_{h}^{3}}\left[\delta\left(\vec{k}^{\prime} \cdot \hat{\vec{u}}_{\vec{k}^{\prime}}\right) \delta\left(\hat{\vec{u}}_{-\vec{k}^{\prime}}-\overline{\overrightarrow{\vec{u}}}_{\vec{k}^{\prime}}\right] \mathrm{d}\{\vec{u}, \vec{X}\}\right.
\end{aligned}
$$

for any Borel set $A$ in the phase space of the system variables, where

$$
\begin{gathered}
\mathbb{Z}_{h}^{3}=\left\{\vec{k} \in \mathbb{Z}^{3}: k_{1}>0 \text { or }\left(k_{1}=0 \text { and } k_{2}>0\right)\right. \\
\text { or } \left.\left(k_{1}=k_{2}=0 \text { and } k_{3}>0\right)\right\}
\end{gathered}
$$

is just "half" the lattice of integers. $Z$ is a normalization constant to make the total probability equal to unity. The delta functions apprearing in the integrand express the constraints on the variables $\left\{\hat{\vec{u}}_{\vec{k}}\right\}_{\vec{k} \in \mathbb{Z}^{3}}$ which result from the fact that they are the Fourier coefficients of a real-valued, incompressible velocity field.

If we substitute the quadratic approximation for the energy into Eq. (20), we deduce that small fluctuations of the system variables $\vec{a}$ about equilbrium are described by Gaussian random variables with mean zero and covariances:

$$
\begin{aligned}
\langle\vec{a} \otimes \overline{\vec{a}}\rangle & \equiv k_{B} T \mathrm{C}, \\
\mathrm{C} & =\left[\begin{array}{cc}
\mathrm{C}^{(f f)} & 0 \\
0 & \mathrm{C}^{(p p)}
\end{array}\right], \\
\mathrm{C}_{\vec{k}, \vec{k}^{\prime}}^{(f f)} & =\left(k_{B} T\right)^{-1}\left\langle\hat{\vec{u}}_{\vec{k}} \otimes \overline{\overrightarrow{\vec{u}}}_{\vec{k}^{\prime}}\right\rangle=M^{-1} \mathrm{P}_{\vec{k}} \delta_{\vec{k}, \vec{k}^{\prime}}, \\
\mathrm{C}_{j, j^{\prime}}^{(p p)} & =\left(k_{B} T\right)^{-1}\left\langle\vec{X}^{(j)} \otimes \vec{X}^{\left(j^{\prime}\right)}\right\rangle=\left[\left(\mathrm{H}^{(0)}\right)^{-1}\right]_{j, j^{\prime}}
\end{aligned}
$$

The Hessian matrix $\mathrm{H}^{(0)}$ is invertible due to the assumption of a nondegenerate, unique energy-minimizing structure configuration. We note that $\mathrm{C}$ is essentially the inverse of the matrix $E$ defining the quadratic energy approximation in Eq. (19); but there are some technical differences due to the constraints relating the Fourier coefficients of the velocity field. In particular, there are two canceling factors of 2 which enter into $C^{(f f)}$ : a factor of 2 in the numerator due to the fact that the Fourier velocity mode $\hat{\vec{u}}_{\vec{k}}$ has a real and imaginary component, and a factor of 2 in the denominator because of the equivalencing of $\hat{\vec{u}}_{\vec{k}}$ and $\overline{\hat{\vec{u}}_{-\vec{k}}}$. These factors of 2 however are absent in both the numerator and denominator for the mode $\vec{k}=\overrightarrow{0}$, which must be real-valued due to the reality condition (12).

The equations (21) express precisely the equipartition theorem for small thermal fluctuations: each degree of freedom of the fluid and the particles has 
energy $\frac{1}{2} k_{B} T$ associated to it.

3.1.0.3 Relation Between Correlation Structure of System Variables and Driving Terms We proceed finally to express the correlation matrix for the system variables in thermal equilibrium in terms of the correlation matrix $\mathrm{G}$ for the driving terms, thereby obtaining a matrix equation for $\mathrm{G}$. From the dynamics (13), we obtain the following evolution for the correlations of the fluctuations of the system variables:

$$
\begin{aligned}
\frac{\mathrm{d}\langle\vec{a}(t) \otimes \overline{\vec{a}(t)}\rangle}{\mathrm{d} t} & =\langle\mathrm{D} \vec{a}(t) \otimes \overline{\vec{a}(t)}\rangle+\langle\vec{a}(t) \otimes \overline{\mathrm{D} \vec{a}(t)}\rangle+\mathrm{G}, \\
& =\mathrm{D}\langle\vec{a}(t) \otimes \overline{\vec{a}(t)}\rangle+\langle\vec{a}(t) \otimes \overline{\vec{a}(t)}\rangle \mathrm{D}^{*}+\mathrm{G} .
\end{aligned}
$$

The last term would be unexpected under the usual product formula for derivatives and arises because the random driving $\vec{g}(t)$ is a function which is "white noise" in time, and therefore incurs stochastic calculus corrections Øksendal (1998). Since the system (13) is linear, there is no ambiguity in how to interpret the white noise; the result (23) follows most directly from the Itô interpretation.

Upon substituting the expression (21a) for the thermal equilibrium covariance structure for the system variables into Eq. (23), we have

$$
0=k_{B} T \mathrm{DC}+k_{B} T \mathrm{CD}^{*}+\mathrm{G}
$$

where $\mathrm{D}^{*}$ denotes the complex conjugate transpose of $\mathrm{D}$. We thereby obtain the following abstract formula for the correlations between the driving of the fluid and particle variables:

$$
\mathrm{G}=-k_{B} T\left(\mathrm{DC}+\mathrm{CD}^{*}\right)
$$

If we were to formally replace $C$ by the inverse of the Hessian of the energy $\mathrm{E}^{-1}$, this statement would be equivalent to one version of the fluctuationdissipation relation (Fox and Uhlenbeck, 1970). We have opted to derive Eq. (24) in a transparent manner because blind application of the usual fluctuation-dissipation relation can run into technical confusions due to the degeneracies in our variables arising from constraints on the Fourier coefficients of the velocity field.

Evaluating now the correlation structure for the thermal driving from Eq. (15) and Eq. (24) for the concrete values of the linearized dynamics matrix D (14d) and system variable covariance matrix C (21) for the IB equations, we have: 


$$
\begin{aligned}
\rho^{-2} \mathrm{P}_{\vec{k}} \mathrm{~F}_{\vec{k}, \vec{k}^{\prime}}^{(f f)} \mathrm{P}_{\vec{k}^{\prime}} & =-k_{B} T\left(\mathrm{D}^{(f f)} \mathrm{C}^{(f f)}+\mathrm{C}^{(f f)} \mathrm{D}^{(f f) *}\right)_{\vec{k}, \vec{k}^{\prime}} \\
& =8 \pi^{2} \frac{k_{B} T \nu|\vec{k}|^{2}}{M L^{2}} \mathrm{P}_{\vec{k}} \delta_{\vec{k}, \vec{k}^{\prime}}, \\
\rho^{-1} \mathrm{P}_{\vec{k}} \mathrm{~F}_{\vec{k}, j^{\prime}}^{(f p)} & =-k_{B} T\left(\mathrm{D}^{(f p)} \mathrm{C}^{(p p)}+\mathrm{C}^{(f f)} \mathrm{D}^{(p f) *}\right)_{\vec{k}, j^{\prime}} \\
& =0 \\
\mathrm{~F}_{j, j^{\prime}}^{(p p)} & =0 .
\end{aligned}
$$

In Eq. (25b), we used the symmetry of $\mathrm{H}^{(0)}$ and the relation $\hat{\delta}_{a, \vec{k}}=\overline{\hat{\delta}_{a, \vec{k}}}$ because $\delta_{a}$ is a real, even function. We therefore deduce that there should be no thermal driving on the particle advection equations $\left(\vec{V}_{r}^{(j)}(t)=0\right.$ for $\left.j=1, \ldots, N\right)$, and that each Fourier mode of the velocity field is to be independently driven by a complex white noise process $\vec{f}_{r, \vec{k}}(t)$ with the amplitudes specified in Eq. (6c). We could of course alter $\vec{f}_{r, \vec{k}}(t)$ by the addition or subtraction of any compressible component parallel to $\vec{k}$ because the pressure term in the Navier-Stokes equation will cancel it. Moreover, some non-Gaussian (generalized) random processes with delta-correlation in time could also serve in place of the Gaus-

sian white noise processes $\tilde{\vec{Z}}_{\vec{k}}(t)$, so long as this random driving was such that a central limit theorem could be invoked to prove that, under the linear evolution (10c), its cumulative effect would be to induce a Gaussian distribution for the system variables in the thermal equilibrium state.

\subsection{Case of Cyclic Coordinates}

We show now how the thermal driving appropriate for a fluid-particle system with a unique, nondegenerate equilibrium state applies as well to the important case in which the system has a family of mechanical equilibria which are related to each other by some symmetry of the particle potential $\Phi$. Following the language of mechanics (Arnold, 1989), we parametrize these symmetries through cyclic coordinates $\boldsymbol{\Xi}_{c}$. Examples of cyclic coordinates are the center of mass and rotational angles of a polymer in the absence of an external potential. Noncyclic coordinates would represent the vibrational modes of the polymer. More precisely, we define a new system of coordinates

$$
\boldsymbol{\Xi}_{c}=\boldsymbol{\Xi}_{c}(\vec{X}), \quad \quad \boldsymbol{\Xi}_{n c}=\boldsymbol{\Xi}_{n c}(\vec{X})
$$

where the sum of the dimensions of the vector of cyclic coordinates $\boldsymbol{\Xi}_{c}$ and the vector of noncyclic coordinates $\boldsymbol{\Xi}_{n c}$ are equal to $N d$, the potential energy depends only on the noncyclic coordinates: $\Phi=\Phi\left(\boldsymbol{\Xi}_{n c}\right)$, and the potential energy has a unique nondegenerate equilibrium $\boldsymbol{\Xi}_{n c}=\boldsymbol{\Xi}_{n c, 0}$ in the reduced 
space of noncyclic coordinates, in the sense that

$$
\left.\mathrm{H}^{(n c, 0)} \equiv \nabla_{\mathbf{\Xi}_{n c}} \boldsymbol{\nabla}_{\mathbf{\Xi}_{n c}} \Phi\right|_{\boldsymbol{\Xi}_{n c}=\boldsymbol{\Xi}_{n c, 0}}
$$

is a strictly positive definite matrix. We moreover assume that the mapping from Euclidean coordinates $\left\{\vec{X}^{(j)}\right\}_{j=1}^{N}$ to the cyclic/noncyclic coordinate decomposition $\left\{\boldsymbol{\Xi}_{n c}, \boldsymbol{\Xi}_{c}\right\}$ is continuously differentiable and locally invertible everywhere. (One has to be careful with cyclic coordinates corresponding to rotation; the standard radial and angle variables have singularities when the radial variable vanishes. But multiplying these standard spherical variables by $r^{2}$, one obtains cyclic coordinates with the desired properties.)

In terms of the new particle coordinates, the IB equations of motion (6) read:

$$
\begin{aligned}
& \frac{\mathrm{d} \boldsymbol{\Xi}_{n c}(t)}{\mathrm{d} t} \\
& =\sum_{j=1}^{N}\left[\vec{u}_{a}\left(\vec{X}^{(j)}\left(\left\{\boldsymbol{\Xi}_{n c}(t), \boldsymbol{\Xi}_{c}(t)\right\}\right)\right)+\vec{V}_{r}^{(j)}(t)\right] \\
& \cdot \nabla_{j} \boldsymbol{\Xi}_{n c}(t), \\
& \frac{\mathrm{d} \boldsymbol{\Xi}_{c}(t)}{\mathrm{d} t} \\
& =\sum_{j=1}^{N}\left[\vec{u}_{a}\left(\vec{X}^{(j)}\left(\left\{\boldsymbol{\Xi}_{n c}(t), \boldsymbol{\Xi}_{c}(t)\right\}, t\right)+\vec{V}_{r}^{(j)}(t)\right]\right. \\
& \cdot \nabla_{j} \boldsymbol{\Xi}_{c}(t) \\
& \rho \frac{\partial \vec{u}(\vec{x}, t)}{\partial t}=\mu \nabla^{2} \vec{u}(\vec{x}, t)-\nabla p(\vec{x}, t) \\
& -\sum_{j=1}^{N} \boldsymbol{\nabla}_{j} \boldsymbol{\Xi}_{n c}(t) \cdot \boldsymbol{\nabla}_{\boldsymbol{\Xi}_{n c}} \Phi\left(\boldsymbol{\Xi}_{n c}(t)\right) \\
& \times \delta_{a}\left(\vec{x}-\vec{X}^{(j)}\left(\left\{\boldsymbol{\Xi}_{n c}(t), \boldsymbol{\Xi}_{c}(t)\right\}\right)\right)+\vec{f}_{r}(\vec{x}, t), \\
& \nabla \cdot \vec{u}(\vec{x}, t)=0 .
\end{aligned}
$$

We now take a Fourier transform of the fluid equations and linearize the fluid velocity and the noncyclic coordinates about their equilibrium values; the cyclic coordinates of course have no equilibrium and cannot be linearized. We thereby obtain: 


$$
\begin{aligned}
& \frac{\mathrm{d} \boldsymbol{\Xi}_{n c}(t)}{\mathrm{d} t}=\sum_{j=1}^{N}\left[\vec{u}_{a}\left(\vec{X}_{0}^{(j)}\left(\boldsymbol{\Xi}_{c}(t)\right), t\right)+\vec{V}_{r}^{(j)}(t)\right] \\
& \cdot \mathrm{J}_{j}^{(n c, 0)}\left(\boldsymbol{\Xi}_{c}(t)\right), \\
& \frac{\mathrm{d} \boldsymbol{\Xi}_{c}(t)}{\mathrm{d} t}=\sum_{j=1}^{N}\left[\vec{u}_{a}\left(\vec{X}_{0}^{(j)}\left(\boldsymbol{\Xi}_{c}(t)\right), t\right)+\vec{V}_{r}^{(j)}(t)\right] \\
& \cdot \nabla_{j} \Xi_{c}(t), \\
& \frac{\partial \hat{\vec{u}}_{\vec{k}}(t)}{\partial t}=-4 \pi^{2} \nu|\vec{k}|^{2} L^{-2} \mathrm{P}_{\vec{k}} \hat{\vec{u}}_{\vec{k}}(t) \\
& -\rho^{-1} \mathrm{P}_{\vec{k}} \sum_{j=1}^{N} \mathrm{~J}_{j}^{(n c, 0)}\left(\boldsymbol{\Xi}_{c}(t)\right) \mathbf{H}^{(n c, 0)} \cdot\left(\boldsymbol{\Xi}_{n c}(t)-\boldsymbol{\Xi}_{n c, 0}\right) \\
& \times \hat{\delta}_{a, \vec{k}} \mathrm{e}^{-2 \pi \mathrm{i} \vec{k} \cdot \vec{X}_{0}^{(j)}\left(\boldsymbol{\Xi}_{c}(t)\right) / L} \\
& +\rho^{-1} \mathrm{P}_{\vec{k}} \vec{f}_{r, \vec{k}}(t),
\end{aligned}
$$

where

$$
\begin{aligned}
\vec{X}_{0}^{(j)}\left(\boldsymbol{\Xi}_{c}\right) & \equiv \vec{X}^{(j)}\left(\left\{\boldsymbol{\Xi}_{n c, 0}, \boldsymbol{\Xi}_{c}\right\}\right), \\
\mathrm{J}_{j}^{(n c, 0)}\left(\boldsymbol{\Xi}_{c}\right) & =\left.\boldsymbol{\nabla}_{j} \boldsymbol{\Xi}_{n c}\right|_{\left\{\vec{X}^{(j)}=\vec{X}_{0}^{(j)}\left(\boldsymbol{\Xi}_{c}\right)\right\}}, \\
\mathbf{H}^{(n c, 0)} & \left.\equiv \boldsymbol{\nabla}_{\boldsymbol{\Xi}_{n c}} \boldsymbol{\nabla}_{\boldsymbol{\Xi}_{n c}} \Phi\left(\boldsymbol{\Xi}_{n c}\right)\right|_{\boldsymbol{\Xi}_{n c}=\boldsymbol{\Xi}_{n c, 0}}
\end{aligned}
$$

Despite the extra complications incurred by the inability to linearize the cyclic coordinates, we can formulate the dynamics in a way which will permit us to generalize our analysis and conclusions from the case of a unique equilibrium state with no cyclic coordinates.

We define a system vector $\vec{a}(t)$ as before, but only include the degrees of freedom of the fluid and the noncyclic coordinates:

$$
\vec{a}(t)=\left[\begin{array}{c}
\left\{\hat{\vec{u}}_{\vec{k}}(t)\right\}_{\vec{k} \in \mathbb{Z}^{3}} \\
\left(\boldsymbol{\Xi}_{n c}(t)-\boldsymbol{\Xi}_{n c, 0}\right)
\end{array}\right] .
$$

Then we can write the linearized dynamics of this system vector in a manner similar to Eq. (13):

$$
\frac{\mathrm{d} \vec{a}(t)}{\mathrm{d} t}=\mathrm{D}\left(\boldsymbol{\Xi}_{c}(t)\right) \vec{a}(t)+\vec{g}(t),
$$

where the terms on the right hand side are defined as follows:

- The linearized dynamics matrix $\mathrm{D}\left(\boldsymbol{\Xi}_{c}\right)$, parametrized by the values of the cyclic coordinates $\boldsymbol{\Xi}_{c}$, can be expressed in block form

$$
\mathrm{D}=\left[\begin{array}{cc}
\mathrm{D}^{(f f)} & \mathrm{D}^{(f p)}\left(\boldsymbol{\Xi}_{c}\right) \\
\mathbf{D}^{(p f)}\left(\boldsymbol{\Xi}_{c}\right) & 0
\end{array}\right]
$$


with the blocks defined:

$$
\begin{aligned}
\mathrm{D}_{\vec{k}, \vec{k}^{\prime}}^{(f f)}= & -4 \pi^{2} \nu|\vec{k}|^{2} L^{-2} \mathrm{P}_{\vec{k}} \delta_{\vec{k}, \vec{k}^{\prime}} \\
\mathrm{D}_{\vec{k}, \cdot}^{(f p)}=- & \rho^{-1} \mathrm{P}_{\vec{k}} \sum_{j=1}^{N} \mathrm{~J}_{j}^{(n c, 0)}\left(\boldsymbol{\Xi}_{c}\right) \mathrm{H}^{(n c, 0)} \hat{\delta}_{a, \vec{k}} \\
& \times \mathrm{e}^{-2 \pi \mathrm{i} \vec{k} \cdot \vec{X}_{0}^{(j)}\left(\Xi_{c}\right) / L}, \\
\mathrm{D}_{\cdot, \vec{k}^{\prime}}^{(p f)}= & L^{3} \hat{\delta}_{a, \vec{k}^{\prime}} \sum_{j=1}^{N} \mathrm{e}^{2 \pi \mathrm{i} \vec{k}^{\prime} \cdot \vec{X}_{0}^{(j)}\left(\boldsymbol{\Xi}_{c}\right) / L} \mathrm{~J}_{j}^{(n c, 0)}\left(\boldsymbol{\Xi}_{c}\right) .
\end{aligned}
$$

A dot in the subscript of a matrix means that the whole range of that index is being simultaneously defined; for example, $\mathrm{D}^{(f p)}$ is defined one full row at a time.

- The thermal driving system vector is defined:

$$
\vec{g}(t)=\left[\begin{array}{c}
\rho^{-1}\left\{\mathrm{P}_{\vec{k}} \vec{f}_{r, \vec{k}}(t)\right\}_{\vec{k} \in \mathbb{Z}^{3}} \\
\sum_{j=1}^{N} \vec{V}_{r}^{(j)}(t) \cdot \mathrm{J}_{j}^{(n c, 0)}\left(\boldsymbol{\Xi}_{c}(t)\right)
\end{array}\right],
$$

with correlation function

$$
\left\langle\vec{g}(t) \otimes \overline{\vec{g}\left(t^{\prime}\right)}\right\rangle=\mathrm{G}\left(\boldsymbol{\Xi}_{c}(t)\right) \delta\left(t-t^{\prime}\right) .
$$

We will want to find what kind of thermal driving $\vec{g}(t)$ induces fluctuations in the fluid and particle variables which are consistent in thermal equilibrium with the Gibbs-Boltzmann distribution (20) which, in terms of the cyclic/noncyclic coordinate system, reads:

$$
\begin{aligned}
& \left.\operatorname{Prob}\left(\left\{\vec{u}, \boldsymbol{\Xi}_{n c}, \boldsymbol{\Xi}_{c}\right\}\right\} \in A\right)= \\
& Z^{-1} \int_{A} \exp \left(-E\left(\vec{u}, \boldsymbol{\Xi}_{n c}\right) /\left(k_{B} T\right)\right) \\
& \prod_{\vec{k} \in \mathbb{Z}_{h}^{3}}\left[\delta\left(\vec{k} \cdot \hat{\vec{u}}_{\vec{k}}\right) \delta\left(\hat{\vec{u}}_{-\vec{k}}-\overline{\hat{\vec{u}}_{\vec{k}}}\right)\right] \\
& \quad \times \mathrm{J}^{-1}\left(\boldsymbol{\Xi}_{n c}, \boldsymbol{\Xi}_{c}\right) \mathrm{d} \vec{u} \mathrm{~d} \boldsymbol{\Xi}_{n c} \mathrm{~d} \boldsymbol{\Xi}_{c}
\end{aligned}
$$

where $Z$ is a normalization factor and $\mathrm{J}^{-1}\left(\boldsymbol{\Xi}_{n c}, \boldsymbol{\Xi}_{c}\right)$ is the inverse of the full Jacobian of the mapping from the Euclidean coordinates $\left\{\vec{X}^{(j)}\right\}_{j=1}^{N}$ to the cyclic and noncyclic coordinates $\left\{\boldsymbol{\Xi}_{n c}, \boldsymbol{\Xi}_{c}\right\}$. Note that this probability distribution is uniform in the cyclic coordinates $\boldsymbol{\Xi}_{c}$ because the energy does not depend on them. Surely the random driving should also respect the symmetry of the potential, and then the dynamics will, under an ergodicity condition, clearly lead to a uniform distribution of the cyclic coordinates, regardless of the detailed nature of the driving. Consistency with the Gibbs-Boltzmann distribution therefore will not prescribe how we should drive the cyclic coordinates. We come back to this point later and proceed with determining how to 
drive the fluid and the noncyclic coordinates in a manner consistent with the Gibbs-Boltzmann distribution for the thermal equilibrium state with small fluctuations of these quantities.

As before, we take a quadratic approximation to the energy to describe weak thermal fluctuations:

$$
\begin{aligned}
E\left(\vec{u}, \boldsymbol{\Xi}_{n c}\right) & \approx E_{\text {app }}\left(\left\{\hat{\vec{u}}_{\vec{k}}\right\}, \boldsymbol{\Xi}_{n c}\right) \\
& =\frac{1}{2} M \sum_{\vec{k} \in \mathbb{Z}^{3}}\left|\hat{\vec{u}}_{\vec{k}}\right|^{2} \\
& +\frac{1}{2}\left(\boldsymbol{\Xi}_{n c}-\boldsymbol{\Xi}_{n c, 0}\right) \cdot \mathrm{H}^{(n c, 0)} \cdot\left(\boldsymbol{\Xi}_{n c}-\boldsymbol{\Xi}_{n c, 0}\right) \\
& \equiv \frac{1}{2} \vec{a} \cdot \mathrm{E} \cdot \overline{\vec{a}}
\end{aligned}
$$

where

$$
\mathrm{E}=\left[\begin{array}{cc}
M \mathrm{I} & 0 \\
0 & \mathrm{H}^{(n c, 0)}
\end{array}\right]
$$

We further replace $\mathbf{J}^{-1}\left(\boldsymbol{\Xi}_{n c}, \boldsymbol{\Xi}_{c}\right)$ in (31) by $\mathbf{J}^{-1}\left(\boldsymbol{\Xi}_{n c, 0}, \boldsymbol{\Xi}_{c}\right)$, upon which we find that small fluctuations of the fluid variables and noncyclic coordinates obey a joint mean zero Gaussian distribution with covariance

$$
\begin{aligned}
\langle\vec{a} \otimes \overrightarrow{\vec{a}}\rangle & \equiv k_{B} T \mathrm{C}, \\
\mathrm{C} & =\left[\begin{array}{cc}
C^{(f f)} & 0 \\
0 & \mathrm{C}^{(p p)}
\end{array}\right] \\
\mathrm{C}_{\vec{k}, \vec{k}^{\prime}}^{(f f)} & =\left(k_{B} T\right)^{-1}\left\langle\hat{\overrightarrow{\vec{u}}}_{\vec{k}} \otimes \overline{\hat{\vec{u}}_{\vec{k}^{\prime}}}\right\rangle=M^{-1} \mathrm{P}_{\vec{k}} \delta_{\vec{k}, \vec{k}^{\prime}}, \\
\mathrm{C}^{(p p)} & =\left(k_{B} T\right)^{-1}\left\langle\left(\boldsymbol{\Xi}_{n c}-\boldsymbol{\Xi}_{n c, 0}\right) \otimes\left(\boldsymbol{\Xi}_{n c}-\boldsymbol{\Xi}_{n c, 0}\right)\right\rangle \\
& =\left(\mathbf{H}^{(n c, 0)}\right)^{-1} .
\end{aligned}
$$

We proceed now as in our first derivation of the fluctuation-dissipation relation (24) for the case of a unique equilibrium, but we replace the thermal averages 
in (23) by conditional averages on a fixed value of $\boldsymbol{\Xi}_{c}$ :

$$
\begin{aligned}
& \frac{\mathrm{d}\left\langle\vec{a}(t) \otimes \overrightarrow{\vec{a}(t)} \mid \boldsymbol{\Xi}_{c}(t)\right\rangle}{\mathrm{d} t}=\left\langle\mathrm{D}\left(\boldsymbol{\Xi}_{c}(t)\right) \vec{a}(t) \otimes \overline{\vec{a}(t)} \mid \boldsymbol{\Xi}_{c}(t)\right\rangle \\
& +\left\langle\vec{a}(t) \otimes \overline{\mathrm{D}\left(\boldsymbol{\Xi}_{c}(t)\right) \vec{a}(t)} \mid \boldsymbol{\Xi}_{c}(t)\right\rangle \\
& +\mathrm{G}\left(\boldsymbol{\Xi}_{c}(t)\right) \\
& +\lim _{t^{\prime} \rightarrow t} \frac{\left\langle\vec{a}(t) \otimes \overrightarrow{\vec{a}(t)} \mid \boldsymbol{\Xi}_{c}\left(t^{\prime}\right)\right\rangle-\left\langle\vec{a}(t) \otimes \overline{\vec{a}(t)} \mid \boldsymbol{\Xi}_{c}(t)\right\rangle}{t^{\prime}-t}, \\
& =\mathrm{D}\left(\boldsymbol{\Xi}_{c}(t)\right)\left\langle\vec{a}(t) \otimes \overline{\vec{a}(t)} \mid \boldsymbol{\Xi}_{c}(t)\right\rangle \\
& +\left\langle\vec{a}(t) \otimes \overline{\vec{a}(t)} \mid \boldsymbol{\Xi}_{c}(t)\right\rangle \mathrm{D}^{*}\left(\boldsymbol{\Xi}_{c}(t)\right) \\
& +\mathrm{G}\left(\boldsymbol{\Xi}_{c}(t)\right) \\
& +\lim _{t^{\prime} \rightarrow t} \frac{\left\langle\vec{a}(t) \otimes \overline{\vec{a}(t)} \mid \boldsymbol{\Xi}_{c}\left(t^{\prime}\right)\right\rangle-\left\langle\vec{a}(t) \otimes \overline{\vec{a}(t)} \mid \boldsymbol{\Xi}_{c}(t)\right\rangle}{t^{\prime}-t} .
\end{aligned}
$$

But according to the Gibbs-Boltzmann distribution (20), the variables $\hat{\vec{u}}_{\vec{k}}$ and $\boldsymbol{\Xi}_{n c}$ which make up the system vector $\vec{a}$ are statistically independent of $\boldsymbol{\Xi}_{c}$ when evaluated simultaneously in a thermal equilibrium state. Consequently, the conditional averages appearing in (33) may be replaced by full thermal averages, which are given by (21). (This argument does not quite imply that $\left\langle\vec{a}(t) \otimes \overrightarrow{\vec{a}(t)} \mid \boldsymbol{\Xi}_{c}\left(t^{\prime}\right)\right\rangle$ can be replaced by its thermal average, because the variables are evaluated at different times, but here one can invoke instead symmetry of the statistics of the noncyclic coordinates with respect to changes in the values of the cyclic coordinates.) We thereby obtain

$$
\mathrm{G}\left(\boldsymbol{\Xi}_{c}(t)\right)=-k_{B} T\left(\mathrm{D}\left(\boldsymbol{\Xi}_{c}(t)\right) \mathrm{C}+\mathrm{CD}^{*}\left(\boldsymbol{\Xi}_{c}(t)\right)\right) .
$$

At this stage, the thermal driving structure matrix $G$ appears like it could depend on the current value of the cyclic coordinate. However, the $\boldsymbol{\Xi}_{c}(t)$ dependence cancels out in the computation:

$$
\mathbf{G}=\left[\begin{array}{cc}
\rho^{-2} \mathrm{PF}^{(f f)} \mathrm{P}^{\dagger} & 0 \\
0 & 0
\end{array}\right],
$$

with the nontrivial block involving the structure of the thermal driving on the fluid having a formula identical to that obtained for the case of a unique equilibrium, Eq. (25a). Consequently, purely driving the fluid with complex white noise according to the prescription in Eq. (6c) is consistent with the GibbsBoltzmann distribution whether or not there are symmetries in the potential energy. Note however when cyclic coordinates exist, it would also be consistent to thermally drive the cyclic particle coordinates by any white noise with correlation structure respecting the symmetry of the potential. The strength of such driving would have to be determined by finer statistical properties, such as the rate of diffusion of the cyclic coordinates. We adopt the simplest strategy of not driving the cyclic particle coordinates at all, which preserves 
the structure of the IB philosophy in that the particles continue to be carried by the fluid. This approach has been shown in Kramer and Peskin (2003); Kramer and Majda (2003/04); Atzberger et al. (2007) to lead to reasonable statistical properties of the cyclic coordinates, though one might contemplate improvements using the freedom to add arbitrary symmetry-preserving driving to the cyclic coordinates.

Finally, we note that the thermal forcing scheme developed will also apply for the case of multiple equilibria (not necessarily related by symmetries). The main reason is that the thermal driving (6) does not actually depend on the local structure of the potential; the scheme is the same regardless of which equilibrium point is used in the linearization.

\section{Thermal Fluctuations for Spatially Discretized Immersed Bound- ary Equations}

In the numerical implementation of the thermally driven immersed boundary equations, one might think of simply discretizing the driving forces in Eq. (6c) by smoothing out the delta-function singularity over a time step $\Delta t$. We endeavor, however, to choose the thermal driving to give an appropriate statistical mechanics for the discretrized IB system. After all, the continuum immersed boundary equations are already an approximation of the true physical equations (for $\Delta x>0$ or $\nu>0$ ) so there is no real difference in philosophy in determining the thermal driving for the discretized immersed boundary equations which are just a further approximation to the physical equations of motion. We are in effect taking the discretized IB equations that are actually being numerically implemented as the governing equations for some physical model, and applying to this system a fluctuation-dissipation argument which will give a precise description for small thermal fluctuations near equilibrium in this physical model. We will consider separately the effects of spatial discretization in this section and the effects of temporal discretization in Section 6.

We therefore begin with the immersed boundary equations, with the standard finite-difference spatial discretization as given in Peskin (2002), as our given equations of motion. The spatial argument will continue to be parameterized by $\vec{x}$, though this variable is restricted to values on the lattice $(\Delta x) \mathbb{Z}_{K}^{3}$, where

$$
\mathbb{Z}_{K}^{3} \equiv[1,2, \ldots, K]^{3}
$$

and $K=L /(\Delta x)$. Spatial derivatives are approximated by finite differences, 
with the following notations:

$$
\begin{aligned}
& \left(D_{\Delta x, m}^{0} \phi\right)(\vec{x})=\frac{\phi\left(\vec{x}+(\Delta x) \hat{\vec{e}}_{m}\right)-\phi\left(\vec{x}-(\Delta x) \hat{\vec{e}}_{m}\right)}{2 \Delta x}, \\
& \vec{D}_{\Delta x}^{0}=\sum_{m=1}^{3} \hat{\vec{e}}_{m} D_{\Delta x, m}^{0}, \\
& L_{\Delta x}^{0}=\sum_{m=1}^{3} \frac{\phi\left(\vec{x}+(\Delta x) \hat{\vec{e}}_{m}\right)-2 \phi(\vec{x})+\phi\left(\vec{x}-(\Delta x) \hat{\vec{e}}_{m}\right)}{(\Delta x)^{2}}
\end{aligned}
$$

note that $L_{\Delta x}^{0}$ is the usual 7-point central-difference Laplace operator.

The spatially discretized IB equations with random thermal driving then read:

$$
\begin{aligned}
\frac{\mathrm{d} \vec{X}^{(j)}(t)}{\mathrm{d} t}= & \sum_{\vec{x} \in(\Delta x) \mathbb{Z}_{K}^{3}} \vec{u}^{n}(\vec{x}) \delta_{a}\left(\vec{x}-\vec{X}^{(j)}(t)\right)(\Delta x)^{3} \\
& +\vec{V}_{r}^{(j)}(t), \\
\frac{\partial \vec{u}(\vec{x}, t)}{\partial t}= & \mu L_{\Delta x}^{0} \vec{u}(\vec{x}, t)-\vec{D}_{\Delta x}^{0} p(\vec{x}, t) \\
& -\sum_{j=1}^{N} \nabla_{j} \Phi\left(\left\{\vec{X}^{(j)}(t)\right\}\right) \delta_{a}\left(\vec{x}-\vec{X}^{(j)}(t)\right) \\
& +\vec{f}_{r}(\vec{x}, t), \\
\vec{D}_{\Delta x}^{0} \cdot \vec{u}(\vec{x}, t)=0 . &
\end{aligned}
$$

The fluctuation-dissipation argument developed for the continuum equation in Section 3, when repeated for this system of equations, yields the following random driving:

$$
\begin{aligned}
\vec{V}_{r}^{(j)}(t) & \equiv 0 \\
\vec{f}_{r}(\vec{x}, t) & =\sum_{\vec{k} \in \mathbb{Z}_{K}^{3}} \vec{f}_{r, \vec{k}}(t) \mathrm{e}^{2 \pi \mathrm{i} \cdot \vec{k} \cdot \vec{x} / L}, \\
\vec{f}_{r, \vec{k}}(t) & =\sqrt{\frac{-\mu k_{B} T \mathcal{F}_{\vec{k}}\left(L_{\Delta x}^{0}\right)}{L^{3}}} \tilde{\vec{Z}}_{\vec{k}}(t) .
\end{aligned}
$$

The change from the continuous-space thermal driving (6c) is the replacement of the amplitude factor $\sqrt{4 \pi^{2} \mu k^{2}}$ by $\sqrt{-\mu \mathcal{F}_{\vec{k}}\left(L_{\Delta x}^{0}\right)}$, where

$$
\mathcal{F}_{\vec{k}}\left(L_{\Delta x}^{0}\right)=\frac{L_{\Delta x}^{0} \mathrm{e}^{2 \pi \mathrm{i} \vec{k} \cdot \vec{x} / L}}{\mathrm{e}^{2 \pi \mathrm{i} \vec{k} \cdot \vec{x} / L}}=-\frac{2}{(\Delta x)^{2}} \sum_{j=1}^{3}\left(1-\cos \frac{2 \pi k_{j}}{K}\right)
$$

is the spectral representation of the finite-difference approximation to the 
Laplacian in the dissipation term. In view of the fluctuation-dissipation relation which, generally speaking, implies that the correlation function of the thermal forcing is proportional to the dissipative terms, this change reflects the change in the viscous dissipation operator from $\mu \nabla^{2}$ to its discretized variant $\mu L_{\Delta x}^{0}$. Note from Eq. (37) that the spectrum of these operators are close to each other for wavenumbers well below the cutoff, $k \ll K$, but that they start to strongly differ for $k \geq K / 2$. In particular, the velocity Fourier modes associated to these high wavenumbers in the discretized IB equations are damped much less strongly than in the continuum limit. If we were to blindly adopt the continuum formulation (6c) for the thermal driving and simply truncate the wavenumbers at the lattice cutoff, the high wavenumbers would be severly overdriven.

\subsection{Derivation of Thermal Driving Terms for Spatially Discretized Equations}

We proceed exactly along the lines described in Section 3; the only differences are that the Stokes equation for the fluid involves finite difference approximations for the spatial derivatives and the Fourier transformed quantities are replaced by their discrete Fourier transform analogues. For example, the fluid degrees of freedom are given by:

$$
\hat{\vec{u}}_{\vec{k}}(t)=\frac{1}{K^{3}} \sum_{\vec{x} \in(\Delta x) \mathbb{Z}_{K}^{3}} \vec{u}(\vec{x}, t) \mathrm{e}^{-2 \pi \mathrm{i} \cdot \vec{k} \cdot \vec{x} / L}
$$

Consequently, in the system of equations (27) allowing for cyclic and noncyclic coordinates, we need only replace $(27 \mathrm{c})$ by:

$$
\begin{aligned}
& \frac{\partial \hat{\vec{u}}_{\vec{k}}(t)}{\partial t}=-\alpha_{\vec{k}} \hat{\vec{u}}_{\vec{k}}(t)+\rho^{-1} \mathrm{P}_{\vec{k}} \vec{f}_{r, \vec{k}}(t), \\
& -\rho^{-1} \mathrm{P}_{\vec{k}} \sum_{j=1}^{N} \mathrm{~J}_{j}^{(n c, 0)}\left(\boldsymbol{\Xi}_{c}(t)\right) \mathbf{H}^{(n c, 0)} \cdot\left(\boldsymbol{\Xi}_{n c}(t)-\boldsymbol{\Xi}_{n c, 0}\right) \\
& \times \mathrm{e}^{-2 \pi \mathrm{i} \vec{k} \cdot \vec{X}_{0}^{(j)}\left(\boldsymbol{\Xi}_{c}(t)\right) / L} \hat{\delta}_{a, \vec{k}}^{(A)},
\end{aligned}
$$

where the Fourier coefficient of the delta function is now aliased due to the lattice wavenumber cutoff:

$$
\hat{\delta}_{a, \vec{k}}^{(A)}=\sum_{\vec{q} \in \mathbb{Z}^{3}} \hat{\delta}_{a, K \vec{q}+\vec{k}}
$$


the projection operator for incompressibility $\mathrm{P}_{\vec{k}}$ is modified to reflect the discretized gradient operator

$$
\begin{gathered}
\mathrm{P}_{\vec{k}}=\mathrm{I}-\frac{\mathcal{F}_{\vec{k}}\left(\vec{D}_{\Delta x}^{0}\right) \otimes \mathcal{F}_{\vec{k}}\left(\vec{D}_{\Delta x}^{0}\right)}{\left|\mathcal{F}_{\vec{k}}\left(\vec{D}_{\Delta x}^{0}\right)\right|^{2}} \\
\mathcal{F}_{\vec{k}}\left(\vec{D}_{\Delta x}^{0}\right)=\frac{\vec{D}_{\Delta x}^{0} \mathrm{e}^{2 \pi \mathrm{i} \vec{k} \cdot \vec{x} / L}}{\mathrm{e}^{2 \pi \mathrm{i} \vec{k} \cdot \vec{x} / L}}=\frac{\mathrm{i}}{\Delta x} \sum_{j=1}^{3} \hat{\vec{e}}_{j} \sin \frac{2 \pi k_{j}}{K}
\end{gathered}
$$

the dissipation factors $\alpha_{\vec{k}}$ now reflects the discretized viscosity term:

$$
\alpha_{\vec{k}}=-\nu \mathcal{F}_{\vec{k}}\left(L_{\Delta x}^{0}\right)
$$

and $\mathcal{F}_{\vec{k}}\left(L_{\Delta x}^{0}\right)$ is defined in Eq. (37).

Upon making the corresponding modifications in the linearized dynamics matrix $\mathrm{D}\left(\boldsymbol{\Xi}_{c}\right)$ in $(30)$, the calculation for the correlations of the thermal driving terms proceeds from here in the same way as for the continuum case worked out in Section 3.

\section{Validity of Simulation Scheme Far From Equilibrium}

The form of the random forces to be introduced in the IB method to simulate thermal fluctuations was based on a linearization of the dynamics about mechanical equilibrium. Many processes in physiology operate far from equilibrium, so an important question is whether the thermal fluctuations simulated by (36) will work well away from equilibrium. This is a rather challenging question to address. In the Stokesian/Brownian dynamics simulation schemes Brady (1993); Brady and Bossis (1988); Ermak and McCammon (1978) which do not explicitly resolve the fluid, the random thermal forces applied to the particles are computed using a fluctuation-dissipation theorem which also applies only for small fluctuations near equilibrium. These same random forces are, however, used in simulations which are very far from equilibrium (for example, in Beard and Schlick (2001)). A possible physical justification for this is that the thermal force just acts additively to whatever systematic forces may be present, so that this thermal force really doesn't depend on the configuration of the system, and the thermal forces applied should be the same both near and far from equilibrium. For this to be true, one must

implicitly be assuming that the system is everywhere in local equilibrium, if not in global equilibrium.

If we accept such an intuitively based justification, we would then be led to ask whether a similar conclusion would apply to random forcing to the fluid. Perhaps the thermal force shouldn't depend on the actual fluid velocity 
configuration, but rather just adds to any systematic forces which are present. The intuitive justification for such a statement might be weaker than for the case of random forcing of a particle, because a fluid is a collective medium rather than a primitive physical object.

Fortunately, a precise result is available for the fluctuations in a pure fluid Español (1998), which indicates that, provided the fluid motion remains incompressible, the only modification needed to generalize the thermal forcing law is to convert Eq. (36) to a physical-space representation and replace the temperature $T$ by the local temperature $T(\vec{x})$ in the physical-space representation of the thermal forcing (36). The fluctuations in local temperature is determined by fluctuations in the local internal energy of the fluid. One would expect that fluctuations in temperature should be negligible compared to its base value of about $300 \mathrm{~K}$ in many microphysiological applications, but we must also note that fluctuations in physical variables become increasingly pronounced as one examines smaller scales. In particular, the local density and local energy density will exhibit more dramatic variations than at the macroscale because the law of large numbers has less power to smooth out deviations in a smaller control volume.

To check whether a constant density and constant temperature approximation remains valid on the microscales of interest, we make some rough quantitative estimates. We will assume the thermal effects are dominant or at least codominant; the smooth component of the motion will be more application dependent and is not expected under typical circumstances to be the source of breakdown of the approximations under consideration. First, incompressibility (which would imply that a constant density approximation should remain good) requires that: $\mathrm{Ma}^{2} \ll \min (\mathrm{Re}, 1)$ (Tritton, 1988) and $\tau \gg \ell / V$ (Landau and Lifshitz, 1987), where $\mathrm{Ma}=V / c$ is the Mach number, $\operatorname{Re}=\ell V / \nu$ is the Reynolds number, $\ell$ is a system length scale, $V$ is a system velocity scale, $\tau$ is a system time scale, $\nu$ is kinematic viscosity, and $c$ is the sound speed. Note that thermal motion sets a particle velocity scale of $\sqrt{k_{B} T /\left(\rho a^{3}\right)}$, which increases with decreasing particle size $a$ ! The grid spacing $h$ will be the smallest length scale resolved in the system, and taking physiological values $c \sim 10^{5} \mathrm{~cm} / \mathrm{s}, T \sim 300 \mathrm{~K}, \rho \sim 1 \mathrm{~g} / \mathrm{cm}^{3}, \nu \sim .01 \mathrm{~cm}^{2} / \mathrm{s}$ along with $k_{B}=1 \times 10^{-16} \mathrm{dyn} / \mathrm{K}$, we find that the constraints on incompressibility require $h \gg\left(\nu^{2} k_{B} T /\left(\rho c^{4}\right)\right)^{1 / 5} \sim 10^{-7} \mathrm{~cm}$ and $\tau \gg \ell / c$. The second criterion will depend on details of the structural dynamics, but if they are comparable to the time scale $\tau \sim \ell^{2} / \nu$ of the fluid dynamics (as might be expected under density-matched conditions), then it will be satisfied on all scales provided it is satisfied on the smallest scale $\ell \sim h$, which in turn requires $h \gg \nu / c \sim 10^{-7} \mathrm{~cm}$. Therefore, incompressibility (and therefore constant density) should hold to a good approximation provided the fluid grid spacing is large compared to $10^{-7} \mathrm{~cm}$, which is required anyway for a continuum representation of the water to make sense. 
To obtain a rough estimate for the size of local temperature fluctuations, we refer to the partial differential equation for the evolution of temperature fluctuations in an incompressible fluid (Landau and Lifshitz, 1987), which has a forcing term proportional to $\nu / c_{P}$ and the square of velocity gradients, a stochastic forcing which scales as $V \sqrt{\nu k_{B} T /\left(\rho c_{P}^{2} \ell^{2}\right)}+\sqrt{\frac{\chi k_{B} T^{2}}{\rho c_{P} \ell^{2}}}$ times a white noise process in space and time (Español, 1998), and a dissipation term $\chi \Delta T$ where $\chi$ is the thermometric conductivity and $c_{P}$ is the specific heat at constant pressure. This will induce temperature fluctuations on the order of

$$
\begin{aligned}
\delta T \sim & \left(\frac{\nu V^{2}}{c_{P} \ell^{2}}\right)\left(\frac{\ell^{2}}{\chi}\right) \\
& +\left(V \sqrt{\frac{\nu k_{B} T}{\rho c_{P}^{2} \ell^{2}}}+\sqrt{\frac{\chi k_{B} T^{2}}{\rho c_{P} \ell^{2}}}\right) \frac{1}{\ell^{3 / 2}}\left(\frac{\ell^{2}}{\chi}\right)^{1 / 2}
\end{aligned}
$$

where we have scaled the deterministic driving linearly and the stochastic driving as the square root of the dissipation time scale $\chi^{-1} \ell^{2}$ and further scaled the white noise in space forcing by $\ell^{-3 / 2}$, corresponding to the resolution length scale $\ell$ of the process. Substituting $c_{P}=4 \times 10^{7} \mathrm{~cm}^{2} /\left(\mathrm{s}^{2} \mathrm{~K}\right)$, $\chi \sim 10^{-3} \mathrm{~cm}^{2} / \mathrm{s}$ (Batchelor, 1967), and other values as above, we find the constraint that $\delta T \ll T$ can be expressed:

$$
h \gg \max \left\{\left(\frac{\nu k_{B}}{\rho \chi c_{P}}\right)^{1 / 3},\left(\frac{k_{B}}{\rho c_{P}}\right)^{1 / 3}\right\} \sim 10^{-7} \mathrm{~cm},
$$

which is the same restriction as for the incompressibility approximation.

Consequently, at least from the order-of-magnitude estimates described above, the thermal forcing scheme developed for the IB method from a near-equilibrium theory can also be expected to be adequate for a wide variety of non-equilibrium simulations under physiological conditions.

The hydrodynamic treatment in Español (1998) does not, however, appear to extend readily in a rigorous manner to the coupling with immersed structures. Here the concern is the general situation in which the thermal fluctuations are strong enough that the nonlinear structure of the particle-interaction forces comes into play. While we have no rigorous justification that our thermal forcing scheme remains precise far from equilibrium in this generality, two formal considerations and one precise calculation indicate that the thermal forcing scheme is either accurate or at least as good as is practically possible in a simulation. First, the same concern about nonlinear particle interactions arises in Stokesian/Brownian dynamics, yet the thermal forces which are applied follow from a near-equilibrium fluctuation-dissipation theorem. Secondly, we have seen in Section 3 that the thermal forcing scheme for the pure fluid is also precisely correct, without change, when the immersed structures are coupled to the fluid. One might therefore hope that the justification from Español 
(1998) for the thermal forcing on the pure fluid, even far from equilibrium under the restrictions noted above, might also imply that the thermal forcing remains appropriate when the immersed structures are coupled in, even with nonlinear fluctuations.

One concrete verification that the simulation scheme is well-behaved even far from equilibrium is that the the probability distribution of the system variables in equilbrium, as simulated by the stochastic IB Method, can be shown to be close to the Gibbs-Boltzmann distribution, without a near-equilbirum assumption. More precisely, the Gibbs-Boltzmann distribution is the exact stationary probabilty distribution for the system variables in the continuum limit. Under spatial discretization, the Gibbs-Boltzmann distribution satisfies the Fokker-Planck equation for the stationary probability distribution up to a small residual which vanishes with the discretization length scale. These results do not imply that the full dynamics of the stochastic IB Method are correct far from equilibrium, but do provide evidence that at least one important statistical law is maintained even far from equilibrium. We next present these calculations.

\subsection{Stationary Distribution Far From Equilibrium}

The probability density $\psi$ for the fluid and structure variables in the stochastic IB method is a time-independent solution of the Fokker-Planck equation associated with the stochastic dynamics, which we will consider with spatial discretization. Working with the Fourier representation of the fluid velocity modes: $\psi=\psi(\hat{\vec{u}}, \vec{X})$, the time-independent Fokker-Planck equation reads

$$
\begin{aligned}
0 & =\mathcal{L}^{*} \psi(\hat{\vec{u}}, \vec{X}) \\
& \equiv-\sum_{j=1}^{N} \nabla_{j} \cdot\left[\sum_{\vec{k} \in \mathbb{Z}_{K}^{3}} L^{3} \mathrm{e}^{2 \pi \mathrm{i} \vec{k} \cdot \vec{X}(j)} / L \hat{\vec{u}}_{\vec{k}} \hat{\delta}_{a, \vec{k}}^{(A)} \psi(\hat{\vec{u}}, \vec{X})\right] \\
& -\sum_{\vec{k} \in \mathbb{Z}_{K}^{3}} \nabla_{\hat{\vec{u}}_{\vec{k}}} \cdot\left[-\alpha_{\vec{k}} \mathrm{P}_{\vec{k}} \hat{\vec{u}}_{\vec{k}} \psi(\hat{\vec{u}}, \vec{X})\right] \\
& -\sum_{\vec{k} \in \mathbb{Z}_{K}^{3}} \nabla_{\hat{\vec{u}}_{\vec{k}}} \cdot\left[\sum_{j=1}^{N}-\mathrm{P}_{\vec{k}} \rho^{-1} \nabla_{j} \Phi(\vec{X}) \hat{\delta}_{a, \vec{k}}^{(A)} \mathrm{e}^{-2 \pi \mathrm{i} \vec{k} \cdot \vec{X}^{(j)} / L}\right. \\
& +\sum_{\vec{k} \in \mathbb{Z}_{K}^{3}} \frac{k_{B} T \alpha_{\vec{k}}}{M} \nabla_{\hat{\vec{u}}_{\vec{k}}} \cdot \nabla_{\overline{\hat{\vec{u}}_{\vec{k}}}} \psi(\hat{\vec{u}}, \vec{X}),
\end{aligned}
$$

where we use the typical convention for the meaning of differentiation with respect to a complex variable as in Kramer and Majda (2003/04). Applying 
the Fokker-Planck operator to the Gibbs-Boltzmann distribution

$$
\psi_{G B}(\hat{\vec{u}}, \vec{X})=\exp \left\{-\frac{1}{k_{B} T}\left[\frac{1}{2} M \sum_{\vec{k} \in \mathbb{Z}_{K}^{3}}\left|\hat{\vec{u}}_{\vec{k}}\right|^{2}+\Phi(\vec{X})\right]\right\},
$$

we obtain

$$
\begin{aligned}
& \mathcal{L}^{*} \psi_{G B}(\hat{\vec{u}}, \vec{X}) \\
& =\sum_{j=1}^{N} \sum_{\vec{k} \in \mathbb{Z}_{K}^{3}}-2 \pi \mathrm{i} \vec{k} \cdot \hat{\vec{u}}_{\vec{k}} L^{2} \mathrm{e}^{2 \pi \mathrm{i} \vec{k} \cdot \vec{X}(j) / L} \psi_{G B}(\hat{\vec{u}}, \vec{X}) .
\end{aligned}
$$

This expression vanishes at least formally in the continuum limit (as then $\vec{k} \cdot \hat{\vec{u}}_{\vec{k}}=0$ for all $\vec{k} \in \mathbb{Z}^{3}$ ), so the Gibbs-Boltzmann distribution is not an exact solution to the Fokker-Planck equation, but has small residual when the fluid mesh spacing is small. This suggests that the stationary probability distribution for the fluid and system variables under the stochastic IB dynamics is approximately of the Gibbs-Boltzmann form, and numerical simulations in Atzberger et al. (2007) confirm this for simple particle systems. A more rig-

orous theoretical formulation of this argument is being prepared for a separate publication by the third author.

\section{Temporal Discretization of Stochastic Immersed Boundary Equa- tions}

Upon passing to a numerical scheme with finite time step $\Delta t$, we must decide how to represent the thermal driving which is delta-correlated in time. Straightforward procedures, such as using the same amplitudes derived in Eq. (36) for continuous time but representing the delta correlations through a $1 / \Delta t$ scaling of the amplitude would only be reasonable if the time step $\Delta t$ is smaller than the relaxation time of all system variables. A more detailed analysis presented in Atzberger et al. (2007); Atzberger and Kramer (2006) provides a numerical representation of the thermal fluctuations which remains accurate even when the time step underresolves some or all of the fluid degrees of freedom. In essence, we exploit the linearity of the fluid equations to derive a stochastic exponential time stepping scheme (Hairer and Lubich, 2000; García-Archilla et al., 1999; Kassam and Trefethen, 2005). The resulting fully discretized numerical algorithm is presented in Section 2.

As shown in Atzberger and Kramer (2006), this numerical method is accurate (in a stochastically strong sense) provided only that the time step $\Delta t$ is much smaller than the time scale over which the immersed structures move a distance comparable to the size $a$ of the elementary particles which are used 
to discretize the structures. That is, the time step must resolve the structural degrees of freedom but need not resolve all (or even any) of the fluid modes. As a detailed discussion of temporal discretization is featured in Atzberger et al. (2007); Atzberger and Kramer (2006), we will not dwell further on this aspect here.

\section{Obstacles with Langevin Dynamics Reformulation of the Im- mersed Boundary Method}

We have used the standard IB equations, in the low Reynolds number limit, as the deterministic system onto which we will add random forces to account for the thermal fluctuations. We explain here why another ostensibly natural approach (Oster and Peskin, 1992) based on Langevin dynamics is not adopted.

A standard self-contained Langevin dynamics model for the position $\vec{X}(t)$ of a particle undergoing Brownian motion in a solvent reads

$$
m \frac{\mathrm{d}^{2} \vec{X}(t)}{\mathrm{d} t^{2}}=-\gamma \frac{\mathrm{d} \vec{X}(t)}{\mathrm{d} t}+\vec{F}(\vec{X}(t), t)+\sqrt{2 \gamma k_{B} T} \vec{Z}(t)
$$

where $m$ is the particle mass, $\gamma$ is a friction coefficient, $\vec{Z}$ represents the white noise thermal forcing, and $\vec{F}(\vec{x}, t)$ describes any forces beyond friction and thermal bombardments with the solvent molecules. This standard equation incorporates thermal forces into the usual macroscopic description by including both a dissipative friction force and a random thermal driving, and this idea has been adopted in many stochastic modeling applications, such as in atmosphere-ocean science (DelSole, 2001; Farrell and Ioannou, 2003; Majda et al., 2001, 2002, 2003, 2006; Franzke et al., 2005).

We consider now how our stochastic modeling framework would appear if we adopt a similar approach (Oster and Peskin, 1992) for the immersed boundary method. Instead of constraining the particles to move at the local fluid velocity (1c), we would then treat each particle as a dynamical entity satisfying Newton's law. We must therefore associate a mass $\left\{m^{(j)}\right\}_{j=1}^{N}$ to each particle, which we could relate to the fluid density multiplied by the effective volume occupied by the $\delta_{a}$ function representing the particle-fluid interaction, if we wish to maintain a constant-density approximation for the system. Writing out Newton's law, we have:

$$
\begin{aligned}
m^{(j)} \frac{\mathrm{d}^{2} \vec{X}^{(j)}(t)}{\mathrm{d} t^{2}}=\gamma^{(j)} & {\left[\vec{u}_{a}\left(\vec{X}^{(j)}(t), t\right)-\frac{\mathrm{d} \vec{X}^{(j)}(t)}{\mathrm{d} t}\right] } \\
& -\nabla_{j} \Phi\left(\left\{\vec{X}^{(j)}(t)\right\}\right)+\vec{F}_{r}^{(j)}(t)
\end{aligned}
$$


where we have included a hydrodynamic force (with drag coefficient $\gamma^{(j)}$ ) in addition to the usual conservative force and a random thermal force $\vec{F}_{r}^{(j)}$.

Note that in allowing the particle to slip relative to the fluid, we have partially broken the IB paradigm of the fluid-particle system as one effective fluid medium. In particular, it is no longer appropriate to state that the forcing term in the Navier-Stokes equation is simply the forces felt by the particles immersed in the fluid. Rather, since the particles are now being treated dynamically, the force applied on the fluid by the particles should be computed using Newton's third law as the force equal and opposite to that which the fluid exerts on the particles. Both the hydrodynamic drag and the random thermal force are such forces acting between the fluid and the particle, so the Stokes equation is recast as follows:

$$
\begin{aligned}
& \rho \frac{\partial \vec{u}(\vec{x}, t)}{\partial t}=\mu \nabla^{2} \vec{u}(\vec{x}, t)-\nabla p(\vec{x}, t)+\vec{f}_{r}(\vec{x}, t) \\
& \quad-\sum_{j=1}^{N}\left\{\gamma^{(j)}\left[\vec{u}_{a}\left(\vec{X}^{(j)}(t), t\right)-\frac{\mathrm{d} \vec{X}^{(j)}(t)}{\mathrm{d} t}\right]+\vec{F}_{r}^{(j)}(t)\right\} \\
& \quad \times \delta_{a}\left(\vec{x}-\vec{X}^{(j)}(t)\right) \\
& \nabla \cdot \vec{u}(\vec{x}, t)=0
\end{aligned}
$$

We have also included an additional thermal force density term $\vec{f}_{r}(\vec{x}, t)$ for the fluid momentum, which we will find to be necessary from statistical mechanical considerations. But first, to make better contact with the usual form of the Stokes equation $5 \mathrm{~b}$ in the IB method, we note that (38b) may be rewritten using (38a) as

$$
\begin{aligned}
& \rho \frac{\partial \vec{u}(\vec{x}, t)}{\partial t}=\mu \nabla^{2} \vec{u}(\vec{x}, t)-\nabla p(\vec{x}, t)+\vec{f}_{r}(\vec{x}, t) \\
& -\sum_{j=1}^{N}\left[\nabla_{j} \Phi\left(\left\{\vec{X}^{(j)}(t)\right\}\right)+m^{(j)} \frac{\mathrm{d}^{2} \vec{X}^{(j)}(t)}{\mathrm{d} t^{2}}\right] \\
& \quad \times \delta_{a}\left(\vec{x}-\vec{X}^{(j)}(t)\right) \\
& \boldsymbol{\nabla} \cdot \vec{u}(\vec{x}, t)=0 .
\end{aligned}
$$

The allowance for the particle to slip by the fluid therefore introduces an extra "inertial force" on the fluid arising from the particle acceleration. We shall refer to the system (38) as the "Immersed Boundary Langevin Particle" System (IBLP system).

By using the fluctuation-dissipation theorem as discussed in Section 3, we find that the appropriate thermal forces on the particles $\left\{\vec{F}_{r}^{(j)}(t)\right\}_{j=1}^{N}$ and on the 
fluid $\vec{f}_{r}(\vec{x}, t)$ for the IBLP system are given as follows (in the continuum limit):

$$
\begin{aligned}
& \vec{F}_{r}^{(j)}(t)=\sqrt{2 \gamma^{(j)} k_{B} T} \vec{Z}^{(j)}(t) \\
& \vec{f}_{r}(\vec{x}, t)=\sum_{\vec{k} \in \mathbb{Z}^{3}} \vec{f}_{r, \vec{k}}(t) \mathrm{e}^{2 \pi \mathrm{i} \vec{k} \cdot \vec{x} / L} \\
& \vec{f}_{r, \vec{k}}(t)=2 \pi k \sqrt{\frac{\mu k_{B} T}{L^{5}}} \tilde{\vec{Z}}_{\vec{k}}(t)
\end{aligned}
$$

where $\left\{\vec{Z}^{(j)}(t)\right\}_{j=1}^{N}$ are real-valued standard white noises in $\mathbb{R}^{3}$ and $\left\{\tilde{\vec{Z}}_{\vec{k}}(t)\right\}_{\vec{k} \in \mathbb{Z}^{3}}$ are complex-valued standard white noises in $\mathbb{C}^{3}$, all of which are mutually independent of one another except for the complex conjugacy relations (7).

Note that it is not physically consistent to omit the random forcing on the fluid. Even if one is interested in the random fluctuations of the particles, these are coupled to the fluid and therefore the fluid must have appropriate thermal fluctuations to give the right statistical properties of the fluctuating particles. The Coupled Langevin Equations approach (Öttinger and Rabin, 1989), which has some structural similarities to the stochastic IB method, also adds thermal driving to both particle and fluid degrees of freedom.

These random forces derived for the IBLP system are straightforward and amenable to efficient simulation. One particularly pleasant feature is that the random forcing on the particles $\vec{F}_{r}^{(j)}(t)$ are independent of one another. In Brownian/Stokesian Dynamics simulation schemes (Brady and Bossis, 1988; Sierou and Brady, 2001; Banchio and Brady, 2003; Ermak and McCammon, 1978; Schlick, 2002) for particles immersed in a fluid, which remove explicit simulation of the fluid from consideration, the thermal forces on the particles are coupled, and the coupling matrix is rather expensive to compute (though significant accelerations have been developed recently (Sierou and Brady, 2001; Banchio and Brady, 2003)), requiring the evaluation of a matrix and its square root every time the particle configuration changes significantly. The fact that the fluid variables are explicitly retained in the IBLP system allows for the appropriate thermal forcing of the particles to be independent of each other.

Unfortunately, while these simple thermal forces are the mathematically correct ones for the IBLP system, this system has some poor physical properties which discommend it as a fundamental model. That is, we have described a fluid-particle system with thermal fluctuations which is mathematically selfconsistent (near equilibrium, at least), but this system is physically ill-founded for reasons we now explore.

\subsection{Physical Inconsistencies of the IBLP System}


7.1.0.4 Constant Friction Coefficient Inconsistent with DensityMatched System Each particle is assumed to have a constant friction coefficient $\gamma^{(j)}$. But even at low Reynolds number, this latter assumption is strictly appropriate only when the fluid momentum relaxes rapidly relative to the particle momentum, which is equivalent to the statement that the fluid density is much less than the particle density (Bocquet and Piasecki, 1997; Deutch and Oppenheim, 1987; Hauge and Martin-Löf, 1973; Masters, 1986; Bocquet, 1998). In fact, even more stringent conditions are necessary when multiple Brownian particles are present, because then the fluid momentum over a length scale comparable to the particle separations must decay faster than the time scale of the particle dynamics (IBc, 1983).

But the IB method, as commonly implemented with Fast Fourier Transform, assumes the whole heterogenous system is at constant density. Even if the particle mass be large compared to the mass of the fluid molecules, the correct description of the evolution of the particle momentum involves the convolution of a hydrodynamic friction function against the past history of the particle's velocity (Deutch and Oppenheim, 1987). That is, the particle's motion is not Markovian due to memory of the history of the particle motion impressed upon the fluid flow in which the particle is moving.

Therefore, we cannot expect that the IBLP system will produce physically meaningful results at least on the short time scales characterizing the relaxation of the particle momentum. Roux (1992), however, has indicated how the long-term dynamics of a fluid-particle system may not be much affected by the misspecification of the frictional effects in a density-matched system by constant coefficients, so long as the system is not interacting on the particle momentum relaxation time scales with chemical or external stimuli. The objection to a constant friction coefficient is therefore not necessarily fatal.

\subsubsection{Hydrodynamic Friction Uses Wrong Fluid Velocity A more} serious physical deficiency with the assumed form of the hydrodynamic drag law in (38a) for its implementation in the IB method is the use of the locally interpolated fluid velocity $\vec{u}_{a}\left(\vec{X}^{(j)}, t\right)$ as a reference velocity for computing the drag. That this is incorrect can be brought out most clearly by focusing attention on a single particle sinking under gravity in a still fluid, where thermal fluctuations can be ignored. Once the particle has reached its terminal velocity, the fluid settles down to a steady state, and the drag force $\vec{F}_{d}$ should clearly just be an effective friction coefficient $\gamma$ of the particle multiplied by the difference between the velocity of the fluid unperturbed by the particle's motion (which here is zero) and the particle's terminal velocity $\vec{U}_{\text {term }}$.

$$
\vec{F}_{d}=-\gamma \vec{U}_{\text {term }}
$$


The IBLP formula for the drag (38a), however, would relate the drag force to the difference between the particle velocity $\vec{U}_{\text {term }}$ and $\vec{u}_{a}(\vec{X}(t), t)$, the velocity of the fluid interpolated over a region of size $a$. The fluid velocity past a particle falling at terminal velocity decays inversely with distance from the particle (Landau and Lifshitz, 1987), so if the particle size were much smaller than the interpolation region length scale, then this would be acceptable. But in the IB implementation, the region of interpolation is precisely the effective particle size, since the forces applied to them are spread to the fluid over a region of this size. Therefore, the reference fluid velocity $\vec{u}_{a}(\vec{X}(t), t)$ that would be used in the drag formula in Eq. (38a) would be on the order of $\left|\vec{U}_{\text {term }}\right|$ instead of zero and would therefore give rise to a grossly underestimated drag force. In particular, a particle falling under gravity would accelerate to much too large a terminal velocity, since the fluid velocity accelerates along with it.

The general problem with the drag force model (38) which causes this bad behavior is as follows. Consider a collection of structures moving due to some external potential and/or interparticle forces without thermal fluctuations. To keep focus on the defect we wish to emphasize, let us suppose for the moment that the system is in a steady flow state, so that the time-independent Stokes equation can be used to describe the fluid velocity. Then the correct way to compute the drag force on a particle is to multiply its constant friction coefficient by the difference between the particle velocity and the velocity the fluid would have at the particle's position if that particle were not present (Auer, 1967). This notion is well-defined in the creeping motion limit because the fluid velocity is instantaneously determined by the particle positions and velocities. The IBLP system's computation of the drag force wrongly subtracts the particle velocity from the fluid velocity interpolated to the particle's position which is of course the fluid velocity which includes the influence of that particle. This physical miscalculation for steady flows clearly indicates discrepancies should also emerge in more time-dependent flow configurations, though the error is more difficult to quantify precisely.

We note that the objections noted here do not apply to the common practice adopted in Stokesian dynamics, in which the drag force for a particle is represented as a friction coefficient multiplied by the difference between the particle velocity and an externally prescribed flow. When the reference velocity field used to calculate the particle drag is not influenced by the particle's motion, then our concern does not apply. Because the IB method intrinsically has the fluid accelerate in response to the forces applied by the immersed structures, however, using a locally interpolated fluid velocity as a reference velocity field for the drag force calculation is problematic. 


\subsection{Some Possible Attempts at Resolution}

One attempt at improving the physical fidelity of the IBLP system would be to try and rectify the misspecification of the drag force in Eq. (38a) at least in the creeping motion (quasi-steady) limit, in which the drag force on a particle can be computed using a constant drag coefficient (Bocquet and Piasecki, 1997; Deutch and Oppenheim, 1987; Hauge and Martin-Löf, 1973; Masters, 1986; Bocquet, 1998). The general idea would be to try to calculate somehow what the fluid velocity at a particle's location would be if that particle were not present, and use that as the reference velocity in the drag law in Eq. (38a) rather than the locally interpolated fluid velocity. Ahlrichs and Dünweg (1999) propose a renormalization of the friction coefficient to accomplish this in a similar context in a lattice-Boltzmann simulation. A related approach would be to try and interpolate the fluid velocity over a larger region in order to mitigate the influence of the particle for which the drag force is being computed. The interpolation length scale would have to be both large enough to neglect the influence of the particle in question but not so large as to also wash out the influence of nearby particles. These ideas might work for dilute systems, but do not seem to generalize nicely when the particles are close enough to be frictionally coupled.

For flows which do not satisfy the creeping motion assumptions, the situation is even more complicated because the notion of a constant friction coefficient isn't even correct as discussed in Paragraph 7.1.0.4.

\subsection{Brownian Dynamics Approach to Immersed Boundary Method}

Another possible resolution is to abandon the Langevin particle dynamics in favor of Brownian particle dynamics. Indeed Kupferman (1996) derived a Brownian particle dynamics scheme through an asymptotic limit of the IBLP equations, and it was this Brownian dynamics which was actually simulated. This would be in close accord with what is done in practice with particle based methods such as Brownian dynamics and Stokesian dynamics (Brady and Bossis, 1988; Sierou and Brady, 2001; Banchio and Brady, 2003; Ermak and McCammon, 1978; Schlick, 2002). We have discussed some deficiencies in the IBLP method, and some of these do persist after the reduction to Brownian dynamics in Kupferman (1996). In particular, one emerges with a random white noise added to the advection equation (1c), some correction terms to the Navier-Stokes equations (1a), and a spurious additional correction term in the advection formula (1c) arising from the improper hydrodynamic drag formula used in the IBLP scheme. 
But perhaps one might try to start afresh with a Brownian dynamics formulation, that is, by adding a random white noise to the particle advection equation (1c) and appropriate terms to the Navier-Stokes equation (1a). Indeed, in the particle-based simulation schemes Brady (1993); Brady and Bossis (1988); Ermak and McCammon (1978), the particle position is generally updated by a Brownian dynamics algorithm, and one could imagine simply using the same thermal forces from those particle-based simulations in the IB equations.

There are, however, a number of questions which would need to be addressed:

- It is not clear that the same thermal particle forces should be used in the IB equations, where the fluid motion is explicitly resolved, as in the schemes where the fluid's effects are replaced by a hydrodynamic friction term.

- It is not clear what terms to add to the Navier-Stokes equations. In particular, should the fluid also be randomly forced? Would this over-diffuse the particles? Without starting from an exact albeit simplified physical model (like both the IB and IBLP systems do), a rational formulation for Brownian dynamics seems a bit elusive.

- The computation of the appropriate random forces on the particles is quite expensive, requiring the evaluation of a $3 N \times 3 N$ matrix and its square root each time the particle configuration changes significantly. This computational challenge arises in conventional Brownian and Stokesian dynamics simulations, though the methods in Sierou and Brady (2001); Banchio and Brady (2003) can significantly accelerate these computations.

\section{Conclusions and Future Work}

We have presented here some further theoretical motivation for the structure of the thermal driving in the stochastic immersed boundary schemes developed in Kramer and Peskin (2003); Kramer and Majda (2003/04); Atzberger et al. (2007), with particular attention to the inclusion of the fluid-structure coupling in the statistical mechanics. Our analysis indicates that the thermal driving scheme corresponds to the theoretically appropriate one prescribed by statistical mechanics, except that the driving of cyclic degrees of freedom (those not constrained by an energetic restoring force) is not actually prescribed by the near-equilbrium analysis. Rather than fluctuating about some mean state, the fluctuations of these modes will grow continuously but unboundedly in time with the character of "Brownian motion" (Reichl, 1998; Kramer, 2005). Theoretical considerations (Kramer and Peskin, 2003; Kramer and Majda, 2003/04; Atzberger et al., 2007) and numerical experiments (Atzberger et al., 2007) indicate that at least the translational Brownian motion of elementary particles is simulated by the stochastic IB method presented in Section 2 in

a physically faithful manner. Moreover, the relative Brownian motion of pairs 
of elementary particles were shown in Kramer and Majda (2003/04) to have many key features accurately represented in the stochastic IB method. According to the statistical mechanical analysis described in the previous sections, it may be appropriate in principle to add additional thermal driving directly on the cyclic degrees of freedom in order to give them the correct diffusivity rates. This would, however, greatly complicated the numerical scheme, requiring in particular a tracking of cyclic and non-cyclic degrees of freedom, as opposed to the present scheme where everything is conducted in standard Euclidean spatial coordinates. We plan to investigate the behavior of more complex cyclic degrees of freedom, such as rotational Brownian motion, under the current scheme and assess whether its statistical properties also remain largely faithful to the laws of statistical physics. As mentioned above, the relative diffusion of particles was shown in Kramer and Majda (2003/04) possibly to lose accuracy at close distances, at least in the sense that the statistical correlations between the particle motion do not quite obey the physical relationships between rigid particles. Other simulation methods (Brady and Bossis, 1988; Sierou and Brady, 2001; Banchio and Brady, 2003; Ermak and McCammon, 1978; Schlick, 2002) generally require special consideration of lubrication forces for particles when they approach each other at close distances, and one avenue for further exploration is the determination of the extent to which these lubrication forces are appropriate in a simulation of flexible structures, and if so, how to incorporate lubrication forces into the Immersed Boundary simulation scheme.

A variety of model systems ranging from polymer knots to a simple molecular motor model have been simulated with quantitative results presented in Atzberger et al. (2007) and movies available at http://www.math.ucsb. edu/ atzberg/stoch_ib/index.html. Improved representations of membranes and more sophisticated molecular motor models are planned for future work. Another direction for methodological development is the incorporation of the stochastic thermal forcing strategy into other variations of the immersed boundary method, such as the immersed interface method (Lee and LeVeque, 2003) and the immersed finite element method (Zhang et al., 2004; Liu et al., 2006).

\section{Acknowledgements}

PRK was partially supported by NSF CAREER Grant DMS-0449717. PJA was partially supported by NSF NSF Mathematical Biology Grant DMS 0635535 . 


\section{References}

C. S. Peskin, The immersed boundary method, Acta Numerica 11 (2002) 479 517.

P. R. Kramer, C. S. Peskin, Incorporating thermal fluctuations into the immersed boundary method, in: K. J. Bathe (Ed.), Computational Fluid and Solid Mechanics 2003, Vol. 2, Elsevier Science Ltd., Oxford, UK, 2003, pp. 1755-1758, proceedings of the Second MIT Conference on Computational Fluid and Solid Mechanics, June 17-20, 2003.

P. R. Kramer, A. J. Majda, Stochastic mode reduction for the immersed boundary method, SIAM J. Appl. Math. 64 (2) (2003/04) 369-400 (electronic).

P. J. Atzberger, P. R. Kramer, C. S. Peskin, A stochastic immersed boundary method for biological fluid dynamics at microscopic length scales, J. Comp. Phys. DOI 10.1016/j.jcp.2006.11.015 (2007).

P. J. Atzberger, A note on the correspondence of an immersed boundary method incorporating thermal fluctuations with Stokesian-Brownian dynamics, Physica D 226 (2) (2007) 144-150, doi:10.1016/j.physd.2006.11.013.

J. F. Brady, G. Bossis, Stokesian dynamics, in: Annual review of fluid mechanics, Vol. 20 of Annu. Rev. Fluid Mech., Annual Reviews, Palo Alto, CA, 1988, pp. 111-157.

A. Sierou, J. F. Brady, Accelerated Stokesian Dynamics simulations, J. Fluid Mech. 448 (2001) 115-146.

A. J. Banchio, J. F. Brady, Accelerated stokesian dynamics: Brownian motion, J. Chem. Phys. 118 (22) (2003) 10323-10332.

D. L. Ermak, J. A. McCammon, Brownian dynamics with hydrodynamic interactions, J. Chem. Phys. 69 (4) (1978) 1352-1360.

T. Schlick, Molecular Modeling and Simulation: An Interdisciplinary Guide, Vol. 21 of Interdisciplinary Applied Mathematics, Springer-Verlag, Berlin, 2002.

J. B. Avalos, A. D. Mackie, Dissipative particle dynamics with energy conservation, Europhys. Lett. 40 (2) (1997) 141-146.

P. Español, Dissipative particle dynamics with energy conservation, Europhys. Lett. 40 (6) (1997) 631-636.

P. Español, P. Warren, Statistical mechanics of dissipative particle dynamics, Europhys. Lett. 30 (4) (1995) 191-196.

P. J. Hoogerbrugge, J. M. V. A. Koelman, Simulating microscopic hydrodynamic phenomena with dissipative particle dynamics, Europhys. Lett. 19 (3) (1992) 155-160.

C. A. Marsh, J. M. Yeomans, Dissipative particle dynamics: The equilibrium for finite time steps, Europhys. Lett. 37 (8) (1997) 511-516.

I. Pagonabarraga, M. H. J. Hagen, D. Frenkel, Self-consistent dissipative particle dynamics algorithm, Europhys. Lett. 42 (4) (1998) 377-382.

R. D. Groot, P. B. Warren, Dissipative particle dynamics: Bridging the gap between atomistic and mesoscopic simulation, J. Chem. Phys. 107 (11) (1997) 
4423-4435.

W. K. Den Otter, J. H. R. Clarke, A new algorithm for dissipative particle dynamics, Europhys. Lett. 53 (4) (2001) 426-431.

P. Nikunen, M. Karttunen, I. Vattulainen, How would you integrate the equations of motion in dissipative particle dynamics simulations?, Computer Physics Communications 153 (3) (2003) 407-423.

A. J. C. Ladd, Numerical simulations of particulate suspensions via a discretized Boltzmann equation. Part 1. Theoretical foundation, J. Fluid Mech. 271 (1994) 285-309.

A. J. C. Ladd, R. Verberg, Lattice-Boltzmann simulations of particle-fluid suspensions, J. Statist. Phys. 104 (5-6) (2001) 1191-1251.

Y. Inoue, Y. Chen, H. Ohashi, Development of a simulation model for solid objects suspended in a fluctuating fluid, J. Statist. Phys. 107 (1/2) (2002) 85-100.

N. Sharma, N. A. Patankar, Direct numerical simulation of the Brownian motion of particles by using fluctuating hydrodynamic equations, J. Comput. Phys. 201 (2004) 466-486.

H. C. Öttinger, Y. Rabin, Diffusion equation versus coupled Langevin equations approach to hydrodynamics of dilute polymer solutions, J. Rheology 33 (5) (1989) 725-743.

P. J. Atzberger, P. R. Kramer, Error analysis of a stochastic immersed boundary method incorporating thermal fluctuations, submitted to Mathematics and Computers in Simulation (2006).

R. Kubo, M. Toda, N. Hashitsume, Statistical physics. II, 2nd Edition, Springer-Verlag, Berlin, 1991, section 4, nonequilibrium statistical mechanics.

R. Kubo, The fluctuation-dissipation theorem, Rep. Prog. Phys. 29 (1966) 255-284.

R. F. Fox, G. E. Uhlenbeck, Contributions to non-equilibrium thermodynamics. I. Theory of hydrodynamical fluctuations, Phys. Fluids 13 (8) (1970) 1893-1902.

R. K. Pathria, Statistical Mechanics, Pergamon Press, Oxford, 1972, section 13.4 .

E. Nelson, Dynamical theories of Brownian motion, Princeton University Press, Princeton, N.J., 1967, Ch. 9.

P. Kramer, Brownian motion, in: A. Scott (Ed.), Encyclopedia of Nonlinear Science, Routledge \& Kegan Paul, London and New York, 2005, pp. 78-81.

L. E. Reichl, A modern course in statistical physics, 2nd Edition, John Wiley \& Sons Inc., New York, 1998, section 5.E.

G. Oster, C. S. Peskin, Dynamics of osmotic fluid flow, in: T. Karalis (Ed.), Swelling Mechanics: From Clays to Living Cells and Tissues, SpringerVerlag, Berlin, 1992, pp. 731-742.

J. F. Brady, Brownian motion, hydrodynamics, and the osmotic pressure, J. Chem. Phys. 98 (4) (1993) 3335-3341.

C. S. Peskin, D. M. McQueen, A general method for the computer simulation 
of biological systems interacting with fluids, in: C. P. Ellington, T. J. Pedley (Eds.), Biological Fluid Dynamics, The Company of Biologists Limited, Cambridge, UK, 1995, pp. 265-276.

J. M. Deutch, I. Oppenheim, The concept of Brownian motion in modern statistical mechanics, in: Brownian Motion, Vol. 83 of Faraday Discuss. Chem. Soc., The Faraday Division of the Royal Society of Chemistry, The Royal Society of Chemistry, London, 1987, pp. 1-20.

R. Glowinski, T. W. Pan, T. I. Hesla, D. D. Joseph, J. Périaux, A fictitious domain approach to the direct numerical simulation of incompressible viscous flow past moving rigid bodies: application to particulate flow, J. Comput. Phys. 169 (2) (2001) 363-426.

N. A. Patankar, P. Singh, D. D. Joseph, R. Glowinski, T.-W. Pan, A new formulation of the distributed Lagrange multiplier/fictitious domain method for particulate flows, International Journal of Multiphase Flow 26 (2000) 1509-1524.

S. J. Karrila, Y. O. Fuentes, S. Kim, Parallel computational strategies for hydrodynamic interactions between rigid particles of arbitrary shape in a viscous fluid, J. Rheology 33 (6) (1989) 913-947.

T. Tran-Cong, N. Phan-Thien, Stokes problems of multiparticle systems: A numerical method for arbitrary flows, Phys. Fluids A 1 (3) (1989) 453-461.

P. J. Atzberger, Velocity correlations of a thermally fluctuating Brownian particle: A novel model of the hydrodynamic coupling, Phys. Lett. A 351 (45) (2006) 225-230.

P. Español, Stochastic differential equations for non-linear hydrodynamics, Physica A 248 (1-2) (1998) 77-96.

A. J. Majda, P. R. Kramer, Simplified models for turbulent diffusion: theory, numerical modelling, and physical phenomena, Phys. Rep. 314 (4-5) (1999) 237-574.

A. M. Yaglom, Correlation theory of stationary and related random functions. Volume I: Basic results, Springer-Verlag, Berlin, 1987.

B. Øksendal, Stochastic differential equations, 5th Edition, Universitext, Springer-Verlag, Berlin, 1998, Ch. 4, an introduction with applications.

V. I. Arnold, Mathematical methods of classical mechanics, 2nd Edition, No. 60 in Graduate Texts in Mathematics, Springer-Verlag, New York, 1989, sections 13,15 .

D. A. Beard, T. Schlick, Computational modeling predicts the structure and dynamics of chromatin fiber, Structure 9 (2) (2001) 105-114.

D. J. Tritton, Physical fluid dynamics, 2nd Edition, Clarendon Press, Oxford, 1988, Ch. 5.8.

L. D. Landau, E. M. Lifshitz, Fluid mechanics, 2nd Edition, Vol. 6 of Course of Theoretical Physics, Pergamon Press, Oxford, 1987, sections 10, 20, 50.

G. K. Batchelor, An introduction to fluid dynamics, Cambridge University Press, Cambridge, U.K., 1967, appendix 1.

E. Hairer, C. Lubich, Long-time energy conservation of numerical methods for oscillatory differential equations, SIAM Journal of Numerical Analysis 
38 (2) (2000) 414-441.

B. García-Archilla, J. M. Sanz-Serna, R. D. Skeel, Long-time-step methods for oscillatory differential equations, SIAM J. Sci. Comput. 20 (3) (1999) 930-963 (electronic).

A.-K. Kassam, L. N. Trefethen, Fourth-order time-stepping for stiff PDEs, SIAM J. Sci. Comput. 26 (4) (2005) 1214-1233 (electronic).

T. DelSole, A theory for the forcing and dissipation in stochastic turbulence models, J. Atmos. Sci. 58 (2001) 3762-3775.

B. F. Farrell, P. J. Ioannou, Structural stability of turbulent jets, J. Atmos. Sci. 60 (2003) 2101-2118.

A. J. Majda, I. Timofeyev, E. Vanden Eijnden, A mathematical framework for stochastic climate models, Comm. Pure Appl. Math. 54 (8) (2001) 891-974.

A. Majda, I. Timofeyev, E. Vanden-Eijnden, A priori tests of a stochastic mode reduction strategy, Phys. D 170 (3-4) (2002) 206-252.

A. Majda, I. Timofeyev, E. Vanden-Eijnden, Systematic strategies for stochastic mode reduction in climate, J. Atmos. Sci. 60 (14) (2003) 1705-1722.

A. Majda, I. Timofeyev, E. Vanden-Eijnden, Stochastic models for selected slow variables in large deterministic systems, Nonlinearity 19 (4) (2006) 769-794.

C. Franzke, A. J. Majda, E. Vanden-Eijnden, Low-order stochastic mode reduction for a realistic barotropic model climate, J. Atmospheric Sci. 62 (6) (2005) 1722-1745.

L. Bocquet, J. Piasecki, Microscopic derivation of non-Markovian thermalization of a Brownian particle, J. Statist. Phys. 87 (5-6) (1997) 1005-1035.

E. H. Hauge, A. Martin-Löf, Fluctuating hydrodynamics and Brownian motion, J. Statist. Phys. 7 (1973) 259-281.

A. J. Masters, Time-scale separations and the validity of the Smoluchowski, Fokker-Planck and Langevin equations as applied to concentrated particle suspensions, Mol. Phys. 57 (2) (1986) 303-317.

L. Bocquet, From a stochastic to a microscopic approach to Brownian motion, Acta Physica Polonica B 29 (6) (1998) 1551-1564.

Concentrated Colloidal Dispersions, Vol. 76 of Faraday Discussions of the Chemical Society, The Faraday Division of the Royal Society of Chemistry, London, 1983, p. 234.

J.-N. Roux, Brownian particles at different times scales: a new derivation of the Smoluchowski equation, Phys. A 188 (4) (1992) 526-552.

P. L. Auer (Ed.), Macromolecules, John Gamble Kirkwood Collected Works, Gordon and Breach Science Publishers, New York, 1967.

P. Ahlrichs, B. Dünweg, Simulation of a single polymer chain in solution by combining lattice Boltzmann and molecular dynamics, J. Chem. Phys. 111 (17) (1999) 8225-8239.

R. Kupferman, Osmotic pressure in confined polymer solutions, notes (Oct. 4 1996).

L. Lee, R. J. LeVeque, An immersed interface method for incompressible Navier-Stokes equations, SIAM J. Sci. Comput. 25 (2003) 832-856. 
URL http://epubs.siam.org/sam-bin/dbq/article/41406

L. Zhang, A. Gerstenberger, X. Wang, W. K. Liu, Immersed finite element method, Comput. Methods Appl. Mech. Engrg. 193 (21-22) (2004) 20512067.

W. K. Liu, Y. Liu, D. Farrell, L. Zhang, X. S. Wang, Y. Fukui, N. Patankar, Y. Zhang, C. Bajaj, J. Lee, J. Hong, X. Chen, H. Hsu, Immersed finite element method and its applications to biological systems, Comput. Methods Appl. Mech. Engrg. 195 (13-16) (2006) 1722-1749. 